\title{
Exact regularized point particle method for multi-phase flows in the two-way coupling regime
}

\author{
P. Gualtieri ${ }^{1}{ }^{*}$ F. Picano ${ }^{2,1}$, G. Sardina ${ }^{3,1}$ \& C.M. Casciola ${ }^{1}$ \\ ${ }^{1}$ Dipartimento di Ingegneria Meccanica e Aerospaziale, \\ Università di Roma La Sapienza Via Eudossiana 18, 00184 Roma Italy. \\ ${ }^{2}$ Linné Flow Center, KTH Mechanics, \\ Osquars Backe 18, SE-100 44 Stockholm, Sweden. \\ ${ }^{3}$ UKE - Università Kore di ENNA \\ Facoltà di Ingegneria, Architettura e Scienze Motorie, \\ Via delle Olimpiadi, 94100 Enna, Italy.
}

April 19, 2022

\begin{abstract}
Particulate flows have been largely studied under the simplifying assumptions of one-way coupling regime where the disperse phase do not react-back on the carrier fluid. For instance, in the context of turbulent flows, many non trivial phenomena such as small scales particles clustering or preferential spatial accumulation have been explained and understood. A more complete view of multiphase flows can be gained calling into play two-way coupling effects, i.e. by accounting for the inter-phase momentum exchange between the carrier and the suspended phase, certainly relevant at increasing mass loading. In such regime, partially investigated in the past by the so-called Particle In Cell (PIC) method, much is still to be learned about the dynamics of the disperse phase and the ensuing alteration of the carrier flow.

In this paper we present a new methodology rigorously designed to capture the inter-phase momentum exchange for particles smaller than the smallest hydrodynamical scale, e.g. the Kolmogorov scale in a turbulent flow. In fact, the momentum coupling mechanism exploits the unsteady Stokes flow around a small rigid sphere where the transient disturbance produced by each particle is evaluated in a closed form. The particles are described as lumped, point masses which would lead
\end{abstract}

*Email address for correspondence: paolo.gualtieri@uniroma1.it 
to the appearance of singularities. A rigorous regularization procedure is conceived to extract the physically relevant interactions between particles and fluid which avoids any "ah hoc" assumption. The approach is suited for high efficiency implementation on massively parallel machines since the transient disturbance produced by the particles is strongly localized in space around the actual particle position. As will be shown, hundred thousands particles can therefore be handled at an affordable computational cost as demonstrated by a preliminary application to a particle laden turbulent shear flow.

\section{Introduction}

Multiphase flows represent the cornerstone of many fields of science and technology ranging from micro-scale devices to the large scale cyclonic separators of industrial plants. In the context of micro/nano technologies, the transport of small particles or bubbles by a carrier fluid is fundamental in designing micro-devices where particles must be separated, mixed or advected towards the sensible regions of the apparatus for detection purposes, see e.g [40]. Concerning larger scale devices, the turbulent transport of a disperse phase is relevant for the dynamics of small fuel droplets in combustion chambers, [36], or in the spatial evolution of sprays employed for surface coating, 33.

Important aspects of multiphase flows are related to the intrinsic coupling between the motion of the disperse phase and the carrier fluid which involves mass, momentum and energy exchange between the two phases. Hydrodynamic interactions among the particles or inter-particles collisions might also occur. The regime where all these interactions take place is known as four-way coupling regime, see e.g. [2, 12. The straightforward method to capture such complex physics is represented by numerical simulations where the fluid flow around each particle is fully resolved. This means that the actual particle boundary has to be resolved on the computational grid and the coupling with the fluid occurs via the non slip boundary conditions imposed on the particle surface. The hydrodynamic force on each particle can be directly computed by integrating the pressure and shear stress distribution on the boundary. Even though this approach captures entirely the physics, it is computationally demanding and limited to the simulation of a relatively small number of "large" particles. The adjective large means that the particle typical size, the diameter $d_{p}$, is larger than the smallest physically active hydrodynamical scale $\eta$. For instance $\eta$ could be either the Kolmogorov dissipative scale in a turbulent flow or the smallest spatial scale 
in a micro-fluidic apparatus. In the context of the so called resolved particles simulations many approaches are available ranging from finite volume techniques, 6], immersed boundary methods, [24], or approaches based on the Lattice-Boltzman equations, 77, 13. Alternative approaches are however available. For instance, the PHYSALIS technique, see e.g. [45] and references therein, has been recently adopted to address the interaction of solid particle and a turbulent flow, [30]. [18] adapted the pseudo-penalization spectral method proposed by [32] to account for the coupled dynamics of neutrally buoyant particles in a turbulent flow. The Force Coupling Method (FCM) proposed by Maxey and coworkers, see e.g. among many others the papers by [26, 23], is certainly worth mentioning in detail. In the FCM the effect that each particle exerts on the fluid is approximated by a multipole expansion of a regularized steady Stokes solution where the concentrated delta-function forces are mollified to a Gaussian. The basic method has been continuously improved by including several physical effects such as lubrication forces for closely packed particles [10] or the effects of elongated particles [22]. Recently a numerical simulation of homogeneous isotropic turbulence laden with thousands of relatively large particles $\left(d_{p} / \eta=6 \div 12\right)$ has been reported by 43 .

The opposite limit of particles much smaller than the smallest hydrodynamical scale is also relevant in many applications. For instance the mixing and combustion of a turbulent spray after that the primary atomization phase has occurred, takes place in presence of significant momentum coupling among the carrier fluid and the fuel droplets, see e.g. the recent review by [19. In fact, in dilute suspensions the volume fraction of the particles is small enough to neglect hydrodynamic interactions and collisions among particles. However, for large values of the particle-to-fluid density ratio, significant mass loads (ratio between the mass of the disperse phase and the fluid) may occur. In such regime, the so called the two-way coupling regime, the momentum exchange between the two phases is significant and must be accounted for. The Particle In Cell (PIC) method, [9], is still a valuable tool to model the momentum coupling. Such approach needs substantial care, however, due to technical issues associated with the injection of the point-wise forcing of the particles on the computational grid where the continuous fluid phase is resolved. Indeed, the force that the particles exert on the fluid is regularized by averaging on the volume of the computational cell. Hence, the coupling term results strongly grid dependent unless the number of particles per cell $N_{p} / N_{c}$ exceeds a certain threshold, see e.g. the numerical results in [17], the discussion by [1] and the comments in [19].

Alternative to the PIC approach, other methods which are able to work 
irrespective of particle number density do indeed exist. For instance [31] modeled the disturbance flow produced by each point particle in terms of the steady Stokeslet. Though interesting, this approach has several potential shortcomings. The disturbance flow decays in space away from the particle as slow as the inverse distance and the perturbation induced by a single particle affects the whole domain. In these conditions, any truncation is undoubtably bound to deeply alter the dynamics. Additionally, the disturbance flow presents the singularity associated with the steady Stokeslet. Moreover, the steady Stokes solution used to model the fluid-particle interaction is not uniformly valid and fails away from the particle. The Oseen correction consistently accounts for the unavoidable far field convective effects, see classical textbooks like [21, 3]. Numerical approaches based on this improved modeling can be found, e.g., in [41, 35.

In the present paper we propose a new approach able to provide a physically consistent and numerically convergent solution for the flow disturbance produced by a huge number of small, massive particles coupled to a generic, possibly turbulent, carrier flow. Hereafter this new formulation will be referred to as the Exact Regularized Point Particle (ERPP) method. As it will be shown in detail, this approach presents several advantages. The most significant one is related to the physical accuracy of the momentum coupling modeling. In a nutshell, in the relative motion with respect to the fluid, the particle generates a vortical field. Even though the relative Reynolds number is small, the local flow is dominated by unsteady viscous effects as discussed by [11]. Vorticity production is a localized process that takes a finite elapsed time $\epsilon_{R}$ since generation to reach the relevant hydrodynamic scales of the flow. It is indeed this transient process of localized generation and finite time diffusion that introduces the actual momentum coupling with the carrier flow. Indeed, the model here envisaged reproduces this physical process by addressing the velocity field, rather than vorticity. The finite time delay $\epsilon_{R}$ automatically provides the regularization of the disturbance field. Instead of being a purely mathematical or numerical ingredient, the regularization featured by ERPP is intrinsically associated with the actual physical process of vorticity generation and viscous diffusion. A distinguishing aspect of ERPP is that all the vorticity generated by the particle is properly transferred to the fluid phase, entailing momentum conservation. A crucial concern is the small scale component of the disturbance field associated with the instantaneously generated vorticity not yet diffused up-to the hydrodynamic scales. This localized, inner scale part of the disturbance exhibits a $1 / r$ local singularity and vanishes altogether at the relevant hydrodynamic scales. Although, in principle, this field should 
contribute locally to the convective term of the Navier-Stokes equations, its effect is proportional to the (small) particle Reynolds number based on the slip velocity. Consistently, it negligibly contributes to the dynamics of the relevant hydrodynamic scales.

Concerning the hydrodynamic force acting on the particles in the two way-coupling regime, the expression provided by [27] is easily adapted to the present context. A crucial issue is the fluid-to-particle slip velocity appearing in the expression of the Stokes drag that should be understood as the undisturbed fluid velocity (i.e. the relative fluid-particle velocity in absence of the particle). In the ERPP the self-induced velocity disturbance can be evaluated in a closed form, allowing to explicitly remove its contribution. It follows a consistent evaluation of slip velocity and hydrodynamic force.

Despite the underlying theoretical aspects may look complicated at first sight, the practical implementation of the ensuing algorithm is remarkably simple and efficient. In principle, the coupling algorithm can indeed be embedded in any available discretization scheme as implemented in one's favorite Navier-Stokes solver. This flexibility allows to easily handle hundred thousands particles at affordable computational cost.

The paper is organized as follows. The next section $\$ 2$ forms the main theoretical body of the paper. Along with its subsections, it introduces the physical model and discusses the inter-phase momentum coupling. In section $\$ 3$ the proposed approach is validated against available analytical results. Section $\$ 4$ reports preliminary results concerning a turbulent particle-laden shear flow. Finally, the last section $\$ 5$ summarizes the main findings. To smooth out the reading, several appendices are devoted to lengthy technical issues whose description inside the main text would have hampered a clear exposition of the main material.

\section{Methodology}

In this section we present the physical model used to achieve the momentum coupling between the carrier fluid and the disperse phase in view of describing the algorithm for the simulation of particle laden flows in the two way coupling regime. In doing so, we assume to know the state of the system at time $t$ and propagate the solution for one time step $D t$. Clearly, reiteration of the procedure allows to proceed in time, as in standard time integration algorithms. During the generic time step of length $D t=t_{n+1}-t_{n}$ the state of the system will propagate from $t_{n}$ to $t_{n+1}$. For the sake of simplicity hereafter we shall often address the generic step as the step $n=0$. In this 
case the running time will be $0 \leq t \leq D t$ in all the differential equations to be addressed. In the discussion, a quantity $\epsilon_{R}$ with dimension of time and the role of a regularization parameter will play a central role. In this case, having dubbed the current time as instant $t_{0}=0$, it could well happen that certain time-delayed variables (i.e. $t-\epsilon_{R}$ ) could be negative: we like to assure the reader before hand that this will be no harm. Integral representation of the solution may represent an exception to this rule. Indeed, such integral representation will be used to derive a systematic regularization procedure from which we obtain by differentiation the regularized pde's to be solved in the algorithm. In such cases the time extrema will typically range in the interval $[0, t]$, with $t$ arbitrary, and typically larger than $\epsilon_{R}$.

In this framework, the short time evolution $(D t \ll 1)$ of the overall flow (fluid and particles) is conceptually split into a (modified) Navier-Stokes evolution of the carrier fluid and a superimposed disturbance flow produced by the relative motion of the particles, here assumed spherical, with respect to the fluid. Relying on the small Reynolds number of the particle-fluid relative motion, the disturbance flow is described by the linear unsteady Stokes equations. In fact, we will rearrange the equation in such a way that the exact solution of the particle disturbance field is consistently embedded into the carrier phase Navier-Stokes solver allowing to reconstruct the actual fluid-particle coupled solution in the limit of vanishing time step and grid spacing for small particle Reynolds number.

The detailed derivation of the coupling model needs a gradual illustration better achieved starting from a schematic description divided in five conceptual steps:

i) Carrier flow-disperse phase interaction and disturbance flow equation (subsection 2.1 )

ii) Solution of the disturbance flow equation (subsection $\$ 2.2$ )

iii) Regularization (subsection \$2.3)

$i v)$ Embedding the disturbance flow into the Navier-Stokes equations (subsection $\$ 2.4$

$v$ ) Evaluation of the hydrodynamic force on the particles in the two-way coupling regime and removal of the self-induced velocity disturbance (subsection $\$ 2.5$ ). 


\subsection{Interaction between the two phases}

In presence of a disperse phase, the carrier fluid fills the domain $\mathcal{D} \backslash \Omega$ where $\mathcal{D}$ is the flow domain and $\Omega(t)=\cup_{p} \Omega_{p}(t)$ denotes the region occupied by the collection of $N_{p}$ rigid particles, with $\Omega_{p}(t)$ the time dependent domain occupied by the $p$ th particle, see the sketch in figure 1. The set theoretic notation $\cup_{p}$ denotes the union of sets indexed by $p$ and $A \backslash B$ denotes the complement in $A$ of set $B$. The motion of the carrier fluid is assumed to be described by the standard incompressible Navier-Stokes equations endowed with the no-slip condition at the particle boundaries

$$
\begin{aligned}
& \left.\begin{array}{l}
\nabla \cdot \mathbf{u}=0 \\
\frac{\partial \mathbf{u}}{\partial t}+\mathbf{u} \cdot \nabla \mathbf{u}=-\frac{1}{\rho_{f}} \nabla \mathbf{p}+\nu \nabla^{2} \mathbf{u}
\end{array}\right\} \quad \quad \mathbf{x} \in \mathcal{D} \backslash \Omega(t) \\
& \left.\mathbf{u}\right|_{\partial \Omega_{p}(t)}=\left.\mathbf{V}_{p}(\mathbf{x})\right|_{\partial \Omega_{p}(t)} \quad p=1, \ldots, N_{p} \\
& \left.\mathbf{u}\right|_{\partial \mathcal{D}}=\mathbf{u}_{\text {wall }} \\
& \mathbf{u}(\mathbf{x}, 0)=\mathbf{u}_{0}(\mathbf{x}) \quad \quad \mathbf{x} \in \mathcal{D} \backslash \Omega(0) .
\end{aligned}
$$

In equations (1), $\mathbf{u}_{0}(\mathbf{x})$ is the velocity field at time $t=0, \rho_{f}$ denotes the fluid density, $\nu$ is the kinematic viscosity, $\partial \Omega_{p}$ is the boundary of the $p$ th particle and $\partial \mathcal{D}$ is the boundary of the overall flow domain, see figure 1 . In this microscopic description, the particles affect the carrier fluid through the noslip condition at the moving particle surface $\partial \Omega_{p}(t)$ where the fluid matches the local rigid body velocity of the particle $\mathbf{V}_{p}(\mathbf{x})=\mathbf{v}_{p}+\boldsymbol{\omega} \times\left(\mathbf{x}-\mathbf{x}_{p}\right)$, with $\mathbf{v}_{p}$ the velocity of the particle geometric center $\mathbf{x}_{p}(t)$ and $\boldsymbol{\omega}(t)$ the angular velocity. The equations of rigid body dynamics need be coupled to the equation for the fluid velocity field to determine the particle motions, where the fluid tension acting at the particle boundary provide the relevant forces and moments.

In principle the system (1) can be numerically integrated at the price of resolving all the particle boundaries on the computational grid. When the suspension is formed by a huge number of small particles their direct solution is unaffordable. In any case, equations (1) still provide the basic description of the flow in terms of the interaction between the two phases. Purpose of the present subsection is to manipulate and approximate the basic equations to derive a viable model for the suspension.

As a starting point, for small time intervals $0 \leq t \leq D t \ll 1$, the carrier flow velocity is decomposed into two parts, $\mathbf{u}(\mathbf{x}, t)=\mathbf{w}+\mathbf{v}$. The field $\mathbf{w}(\mathbf{x}, t)$ 


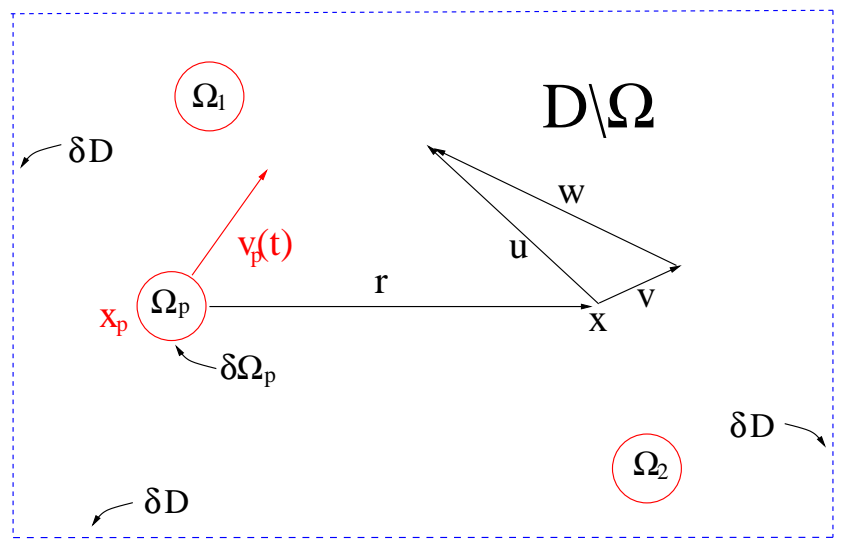

Figure 1: Sketch of the flow domain. The fluid fills the domain $\mathcal{D} \backslash \Omega$ with $\Omega(t)=\cup_{p} \Omega_{p}(t)$ the region occupied by the $N_{p}$ rigid particles and $\Omega_{p}(t)$ the time dependent domain of the $p$ th particle. $\partial \mathcal{D}$ denotes the boundary of $\mathcal{D}$. The fluid velocity at the generic point $\mathbf{x} \in \mathcal{D} \backslash \Omega$ is decomposed as $\mathbf{u}=\mathbf{w}+\mathbf{v}$, to be understood as the definition of $\mathbf{w}$ given the fluid velocity $\mathbf{u}$ and the solution $\mathbf{v}$ of the linear, unsteady Stokes problem (4).

is assumed to satisfy the equations

$$
\begin{aligned}
& \nabla \cdot \mathbf{w}=0 \\
& \frac{\partial \mathbf{w}}{\partial t}+\mathbf{F}=-\frac{1}{\rho_{f}} \nabla \pi+\nu \nabla^{2} \mathbf{w} \\
& \left.\mathbf{w}\right|_{\partial \mathcal{D}}=\mathbf{u}_{\text {wall }}-\mathbf{v}_{\partial \mathcal{D}} \\
& \mathbf{w}(\mathbf{x}, 0)=\overline{\mathbf{u}}_{0}(\mathbf{x})
\end{aligned}
$$

where $\mathbf{x} \in \mathcal{D}$ and

$$
\mathbf{F}= \begin{cases}\mathbf{u} \cdot \nabla \mathbf{u} & \text { for } \mathbf{x} \in \mathcal{D} \backslash \Omega(t) \\ \mathbf{V}_{p} \cdot \nabla \mathbf{V}_{p} & \text { for } \mathbf{x} \in \Omega(t)\end{cases}
$$

is a field reproducing the complete convective term of the Navier-Stokes equation in the carrier fluid domain $\mathcal{D} \backslash \Omega$ which is prolonged inside $\Omega$ using the solid particle velocity field. Other choices are possible, but the actual shape of the field inside the particle domains is irrelevant to our present purposes: under this respect, the solid body motion provides an elegant example given the continuity of the field $\mathbf{F}$ at the particle boundaries. In 
problem (2), a part from the prolongation of the field $\mathbf{F}$, the particles disappeared altogether from the domain and the convective term, retaining its complete nonlinear nature in the fluid domain, is treated as a prescribed forcing term. The initial field $\overline{\mathbf{u}}_{0}$ is prolonged inside the particle domains by the same rule, i.e. as the solid body motion of relevant particle.

The field $\mathbf{v}(\mathbf{x}, t)$ exactly satisfies the linear unsteady Stokes problem (the complete non-linear term has been retained in the equation for $\mathbf{w}$ )

$$
\begin{gathered}
\nabla \cdot \mathbf{v}=0 \\
\left.\begin{array}{c}
\frac{\partial \mathbf{v}}{\partial t}=-\frac{1}{\rho_{f}} \nabla q+\nu \nabla^{2} \mathbf{v}
\end{array}\right\} \quad \mathbf{x} \in \mathcal{D} \backslash \Omega(t) \\
\left.\mathbf{v}\right|_{\partial \Omega_{p}(t)}=\left.\mathbf{V}_{p}(\mathbf{x})\right|_{\partial \Omega_{p}(t)}-\left.\mathbf{w}\right|_{\partial \Omega_{p}(t)} \quad p=1, \ldots N_{p} \\
\mathbf{v}(\mathbf{x}, 0)=0 \quad \mathbf{x} \in \mathcal{D} \backslash \Omega(0),
\end{gathered}
$$

where boundary conditions are applied at the particle surfaces. It should be observed that no boundary condition are applied to the field $\mathbf{v}$ at the flow domain boundary $\partial \mathcal{D}$. In other words, the field $\mathbf{v}$ can be regarded as a free space solution in the whole $\mathbb{R}^{3}$ restricted the actual flow domain $\mathcal{D}$. Indeed the value of $\mathbf{v}$ at the domain boundary is used to correct the boundary condition for $\mathbf{w}$. It is worth calling the reader's attention to the initial conditions for the two complementary problems: the initial velocity field is assigned as initial condition for $\mathbf{w}$, leaving homogenous initial data for $\mathbf{v}$. As shown in a later section, the homogeneous initial conditions for the perturbation field $\mathbf{v}$ will turn out to be a crucial feature of the decomposition.

The solution of equations (4) can be expressed in terms of the boundary integral representation of the unsteady Stokes equations that involves the unsteady Stokeslet $G_{i j}(\mathbf{x}, \boldsymbol{\xi}, t, \tau)$, a second order Cartesian tensor, and the associated stresses in the form of the third order tensor $\mathcal{T}_{i j k}(\mathbf{x}, \boldsymbol{\xi}, t, \tau)$, see appendix A.2 and classical textbooks, [44, 20]. The unsteady Stokeslet $G_{i j}(\mathbf{x}, \boldsymbol{\xi}, t, \tau)$ is readily interpreted as the fluid velocity (ith direction) at position $\mathbf{x}$ and time $t$ due to the singular forcing $\delta(\mathbf{x}-\boldsymbol{\xi}) \delta(t-\tau)(j$ th direction) applied at $\boldsymbol{\xi}$ at time $\tau$. Exploiting the vanishing initial condition, the solution of equations (4) is recast in the boundary integral representation

$$
v_{i}(\mathbf{x}, t)=\int_{0}^{t} d \tau \int_{\partial \Omega} t_{j}(\boldsymbol{\xi}, \tau) G_{i j}(\mathbf{x}, \boldsymbol{\xi}, t, \tau)-v_{j}(\boldsymbol{\xi}, \tau) \mathcal{T}_{i j k}(\mathbf{x}, \boldsymbol{\xi}, t, \tau) n_{k}(\boldsymbol{\xi}) d S_{\boldsymbol{\xi}}
$$

Equation (5) expresses $\mathbf{v}(\mathbf{x}, t)$ in terms of a boundary integral on $\partial \Omega=$ $\cup_{p} \partial \Omega_{p}$ involving the (physical) tension $t_{j}(\boldsymbol{\xi}, \tau)$ and the boundary condition 
on the perturbation velocity $v_{j}(\boldsymbol{\xi}, \tau)$ at each particle boundary. In principle, the tension $t_{j}(\boldsymbol{\xi}, \tau)$ can be determined by solving the boundary integral equation (indeed a system of coupled boundary integral equations, one for each particle) associated with representation (5). Once the tension is known at each particle boundary, representation (5) provides the perturbation field everywhere in the flow domain. Moreover the boundary integral of the tension $t_{j}$ would provide the forces acting on the particles.

Since the present aim is capturing the effects of many small particles of diameter $d_{p}$, the interest is focused on the far field particle disturbance that can be approximated by a multipole expansion of equation (5). Substituting in equation (5) the first order truncation of the Taylor series of $G_{i j}(\mathbf{x}, \boldsymbol{\xi}, t, \tau)$ and $\mathcal{T}_{i j k}(\mathbf{x}, \boldsymbol{\xi}, t, \tau)$, centered at the particle position $\mathbf{x}_{p}$, leads to the far field expression for large $r_{p} / d_{p}$, where $r_{p}=\left|\mathbf{x}-\mathbf{x}_{p}\right|$,

$$
v_{i}(\mathbf{x}, t)=-\sum_{p} \int_{0}^{t} D_{j}^{p}(\tau) G_{i j}\left(\mathbf{x}, \mathbf{x}_{p}, t, \tau\right) d \tau
$$

showing that the far field disturbance depends only on the hydrodynamic force $\mathbf{D}_{p}(\tau)$, with Cartesian components $D_{j}^{p}$, which acts on the generic particle. Given the physical interpretation of the unsteady Stokeslet $G_{i j}$, the partial differential equation whose solution is given by (6) follows as

$$
\frac{\partial \mathbf{v}}{\partial t}-\nu \nabla^{2} \mathbf{v}+\frac{1}{\rho_{f}} \nabla \mathbf{q}=-\frac{1}{\rho_{f}} \sum_{p} \mathbf{D}_{p}(t) \delta\left[\mathbf{x}-\mathbf{x}_{p}(t)\right] ; \quad \mathbf{v}(\mathbf{x}, 0)=0
$$

as is directly verified by combining the time derivative of equation (6) with its Laplacian. In equation (7) the boundary condition at the particle surfaces disappear altogether and the fluid-particle coupling occurs via the (singular) forcing term in the unsteady Stokes problem. Given the linearity, hereafter we shall explicitly consider the single contribution of the generic particle $p$, keeping in mind that a final summation all over the particles is required.

It is also clear that as the particle diameter gets smaller and smaller, the term $\mathbf{F}$ in equations (2) uniformly fills the entire domain $\mathcal{D}$ and reduces everywhere to the standard convective term of the Navier-Stokes equation $\mathbf{u} \cdot \nabla \mathbf{u}$, where $\mathbf{u}=\mathbf{w}+\mathbf{v}$.

\subsection{Disturbance flow due to a small particle}

The vorticity equation associated with (7) is

$$
\frac{\partial \boldsymbol{\zeta}}{\partial t}-\nu \nabla^{2} \boldsymbol{\zeta}=\frac{1}{\rho_{f}} \mathbf{D}_{p}(t) \times \nabla \delta\left[\mathbf{x}-\mathbf{x}_{p}(t)\right] ; \quad \boldsymbol{\zeta}(\mathbf{x}, 0)=0
$$


where $\boldsymbol{\zeta}=\nabla \times \mathbf{v}$. The solution can be expressed as a convolution with the fundamental solution of the diffusion equation $g(\mathbf{x}-\boldsymbol{\xi}, t-\tau)$, given by (see appendix A.1)

$$
g(\mathbf{x}-\boldsymbol{\xi}, t-\tau)=\frac{1}{[4 \pi \nu(t-\tau)]^{3 / 2}} \exp \left[-\frac{\|\mathbf{x}-\boldsymbol{\xi}\|^{2}}{4 \nu(t-\tau)}\right],
$$

that is a Gaussian with time dependent variance $\sigma(t-\tau)=\sqrt{2 \nu(t-\tau)}$. Observe that $g$ is the fundamental solution of the diffusion equation in freespace, since $\mathbf{v}$ is itself a free-space field, as noted when discussing eq. (4).

By rearranging the forcing on the right hand side of equation (8) as a time-convolution,

$$
\left.\mathbf{D}_{p}(t) \times \nabla \delta\left[\mathbf{x}-\mathbf{x}_{p}(t)\right]=\int_{0}^{t^{+}} \mathbf{D}_{p}(\tau) \times \nabla \delta\left[\mathbf{x}-\mathbf{x}_{p}(\tau)\right] \delta(t-\tau) d \tau\right]
$$

the solution of equation (8) follows at once as

$$
\boldsymbol{\zeta}(\mathbf{x}, t)=\frac{1}{\rho_{f}} \int_{0}^{t^{+}} \mathbf{D}_{p}(\tau) \times \nabla g\left[\mathbf{x}-\mathbf{x}_{p}(\tau), t-\tau\right] d \tau .
$$

The original fluid velocity $\mathbf{v}(\mathbf{x}, t)$ can be reconstructed from the vorticity using the non-canonical decomposition

$$
\mathbf{v}(\mathbf{x}, t)=\mathbf{v}_{\boldsymbol{\zeta}}(\mathbf{x}, t)+\nabla \phi(\mathbf{x}, t)
$$

where

$$
\mathbf{v}_{\boldsymbol{\zeta}}(\mathbf{x}, t)=-\frac{1}{\rho_{f}} \int_{0}^{t^{+}} \mathbf{D}_{p}(\tau) g\left[\mathbf{x}-\mathbf{x}_{p}(\tau), t-\tau\right] d \tau
$$

is a pseudo-velocity, such that its curl equals the vorticity, $\nabla \times \mathbf{v}_{\boldsymbol{\zeta}}=\boldsymbol{\zeta}$, and the gradient term is added to make the field solenoidal, as appropriate for incompressible flows,

$$
\nabla^{2} \phi(\mathbf{x}, t)=-\frac{1}{\rho_{f}} \int_{0}^{t^{+}} \mathbf{D}_{p}(\tau) \cdot \nabla g\left[\mathbf{x}-\mathbf{x}_{p}(\tau), t-\tau\right] d \tau .
$$

The pseudo-velocity $\mathbf{v}_{\boldsymbol{\zeta}}$ obeys the equation

$$
\begin{aligned}
\frac{\partial \mathbf{v}_{\boldsymbol{\zeta}}}{\partial t}-\nu \nabla^{2} \mathbf{v}_{\boldsymbol{\zeta}}=-\frac{1}{\rho_{f}} \mathbf{D}_{p}(t) \delta\left[\mathbf{x}-\mathbf{x}_{p}(t)\right], \quad \mathbf{v}_{\boldsymbol{\zeta}}(\mathbf{x}, 0)=0 . \\
{ }^{1} \text { with } \int_{0}^{t^{+}} f(\tau) d \tau \text { we intend } \lim _{\epsilon \rightarrow 0} \int_{0}^{t+\epsilon} f(\tau) d \tau
\end{aligned}
$$




\subsection{Regularization of the disturbance field due to a small particle}

Both the velocity $\mathbf{v}$ and the vorticity $\boldsymbol{\zeta}$ are apparently singular, with singularity arising from the contribution to the integral near the upper integration limit, $\tau \simeq t$, where $g(\mathbf{x}-\boldsymbol{\xi}, t-\tau)$ tends to behave as "badly" as the Dirac delta function. On the contrary away from the upper integration limit the integrand is nicely behaved since it involves a Gaussian or its gradient.

In this paragraph we define a regularization procedure based on a temporal cut-off $\epsilon_{R}$ such that the fields are additively split into a regular and a singular component. For instance the decomposition of the vorticity reads

$$
\boldsymbol{\zeta}(\mathbf{x}, t)=\boldsymbol{\zeta}_{R}\left(\mathbf{x}, t ; \epsilon_{R}\right)+\boldsymbol{\zeta}_{S}\left(\mathbf{x}, t ; \epsilon_{R}\right),
$$

with smooth and singular part respectively given by

$$
\boldsymbol{\zeta}_{R}(\mathbf{x}, t)=\frac{1}{\rho_{f}} \int_{0}^{t-\epsilon_{R}} \mathbf{D}_{p}(\tau) \times \nabla g\left[\mathbf{x}-\mathbf{x}_{p}(\tau), t-\tau\right] d \tau,
$$

and by

$$
\boldsymbol{\zeta}_{S}(\mathbf{x}, t)=\frac{1}{\rho_{f}} \int_{t-\epsilon_{R}}^{t^{+}} \mathbf{D}_{p}(\tau) \times \nabla g\left[\mathbf{x}-\mathbf{x}_{p}(\tau), t-\tau\right] d \tau .
$$

As implied by the fundamental solution of the diffusion equation, the regular part of the vorticity field is everywhere smooth and characterized by the smallest spatial scale $\sigma_{R}=\sigma\left(\epsilon_{R}\right)=\sqrt{2 \nu \epsilon_{R}}$. Thanks to the semigroup property of solutions of the diffusion equation, the regular field $\boldsymbol{\zeta}_{R}(\mathbf{x}, t)$ can be interpreted as the free diffusion from time $t-\epsilon_{R}$ to time $t$ of the complete field at time $t-\epsilon_{R}, \boldsymbol{\zeta}\left(\mathbf{x}, t-\epsilon_{R}\right)$, namely

$$
\boldsymbol{\zeta}_{R}(\mathbf{x}, t)=\int \boldsymbol{\zeta}\left(\boldsymbol{\xi}, t-\epsilon_{R}\right) g\left(\mathbf{x}-\boldsymbol{\xi}, \epsilon_{R}\right) d \boldsymbol{\xi}
$$

where the spatial convolution integral propagates the field from $t-\epsilon_{R}$ to $t$. Although physically obvious, equation $(19)$ can be directly proved using the result

$$
g(\mathbf{x}, t)=\int g\left(\boldsymbol{\xi}, t-\epsilon_{R}\right) g\left(\mathbf{x}-\boldsymbol{\xi}, \epsilon_{R}\right) d \boldsymbol{\xi},
$$

that is nothing more that a re-expression of the semigroup property for the free-space diffusion equation applied to the fundamental solution $g$. Actually, using the property (20) and introducing eq. (11) at time $t-\epsilon_{R}$ into 
eq. (19), after integration by parts, one readily gets

$$
\begin{array}{r}
\boldsymbol{\zeta}_{R}(\mathbf{x}, t)=\frac{1}{\rho_{f}} \int\left\{\int_{0}^{t-\epsilon_{R}} \mathbf{D}_{p}(\tau) \times \nabla_{\boldsymbol{\xi}} g\left[\boldsymbol{\xi}-\mathbf{x}_{p}(\tau), t-\epsilon_{R}-\tau\right] d \tau\right\} g\left(\mathbf{x}-\boldsymbol{\xi}, \epsilon_{R}\right) d \boldsymbol{\xi}= \\
\frac{1}{\rho_{f}} \int_{0}^{t-\epsilon_{R}} \mathbf{D}_{p}(\tau) \times \int \nabla_{\boldsymbol{\xi}} g\left[\boldsymbol{\xi}-\mathbf{x}_{p}(\tau), t-\epsilon_{R}-\tau\right] g\left(\mathbf{x}-\boldsymbol{\xi}, \epsilon_{R}\right) d \boldsymbol{\xi} d \tau= \\
\frac{1}{\rho_{f}} \int_{0}^{t-\epsilon_{R}} \mathbf{D}_{p}(\tau) \times \nabla \int g\left[\boldsymbol{\xi}-\mathbf{x}_{p}(\tau), t-\epsilon_{R}-\tau\right] g\left(\mathbf{x}-\boldsymbol{\xi}, \epsilon_{R}\right) d \boldsymbol{\xi} d \tau= \\
\frac{1}{\rho_{f}} \int_{0}^{t-\epsilon_{R}} \mathbf{D}_{p}(\tau) \times \nabla g\left[\mathbf{x}-\mathbf{x}_{p}(\tau), t-\tau\right] d \tau,
\end{array}
$$

which is indeed equation (17). The corresponding vorticity field $\boldsymbol{\zeta}_{R}$ at time $t$ obeys a forced diffusion equation where the forcing is applied at the slightly earlier time $t-\epsilon_{R}$,

$\frac{\partial \boldsymbol{\zeta}_{R}}{\partial t}-\nu \nabla^{2} \boldsymbol{\zeta}_{R}=-\frac{1}{\rho_{f}} \nabla \times \mathbf{D}_{p}\left(t-\epsilon_{R}\right) g\left[\mathbf{x}-\mathbf{x}_{p}\left(t-\epsilon_{R}\right), \epsilon_{R}\right] ; \quad \boldsymbol{\zeta}_{R}(\mathbf{x}, 0)=0$,

see appendix A.3 for the detailed calculation. The velocity field $\mathbf{v}_{R}$ associated with the regularized vorticity field $\boldsymbol{\zeta}_{R}$ can be expressed though the general decomposition 12 ,

$$
\mathbf{v}_{R}(\mathbf{x}, t)=\mathbf{v}_{\boldsymbol{\zeta}_{R}}+\nabla \Phi_{R}
$$

where, by analogy with eq. 15, , the regularized pseudo-velocity $\mathbf{v}_{\boldsymbol{\zeta}_{R}}$ is

$\frac{\partial \mathbf{v}_{\boldsymbol{\zeta}_{R}}}{\partial t}-\nu \nabla^{2} \mathbf{v}_{\boldsymbol{\zeta}_{R}}=-\frac{1}{\rho_{f}} \mathbf{D}_{p}\left(t-\epsilon_{R}\right) g\left[\mathbf{x}-\mathbf{x}_{p}\left(t-\epsilon_{R}\right), \epsilon_{R}\right] ; \quad \mathbf{v}_{\boldsymbol{\zeta}_{R}}(\mathbf{x}, 0)=0$,

and the potential correction follows from the equation

$$
\nabla^{2} \Phi_{R}=-\nabla \cdot \mathbf{v}_{\boldsymbol{\zeta}_{R}}
$$

It is worth noticing that the complete regularized field obeys instead the forced unsteady Stokes equation

$$
\frac{\partial \mathbf{v}_{R}}{\partial t}-\nu \nabla^{2} \mathbf{v}_{R}+\frac{1}{\rho_{f}} \nabla \mathrm{q}_{R}=-\frac{1}{\rho_{f}} \mathbf{D}_{p}\left(t-\epsilon_{R}\right) g\left[\mathbf{x}-\mathbf{x}_{p}\left(t-\epsilon_{R}\right), \epsilon_{R}\right]
$$

for the solenoidal field $\mathbf{v}_{R}$. The crucial point to observe here is that the regularized component of the velocity disturbance $\mathbf{v}_{R}(\mathbf{x}, t)$ evolves according to a diffusion equation forced by the anticipating Stokes drag (i.e. evaluated 
at $\left.t-\epsilon_{R}\right)$ times the regular spatial distribution $g\left[\mathbf{x}-\mathbf{x}_{p}\left(t-\epsilon_{R}\right), \epsilon_{R}\right]$. Equation (23) can in principle be straightforwardly solved on a discrete grid, once the spatial scale $\sigma_{R}$ of the forcing is properly resolved by the grid. Once $\mathbf{v}_{\boldsymbol{\zeta}_{R}}$ is known, the correction needed to make the field solenoidal calls for the solution of the Poisson equation (24).

For the future application to the full solver for the carrier phase in presence of the suspension, it is also worth mentioning that the field $\mathbf{v}_{\boldsymbol{\zeta}_{R}}$ is rapidly decaying in space as far as the observation time $t$ is small, since it implies the short-time diffusion of a rapidly decaying forcing. All the longrange effects are indeed confined to the potential correction $\nabla \Phi_{R}$. As will be discussed in the forthcoming sections, the field $\mathbf{v}_{R}$ does not need to be separately evaluated, since it will be embedded in the solution procedure for the single field $\mathbf{u}$ which accounts for both the undisturbed carrier flows and the particle perturbation.

At variance with $\mathbf{v}_{R}$, the singular contribution $\mathbf{v}_{S}$ cannot be represented on a discrete grid. It can be decomposed as well into a vorticity related component plus a potential correction, according to the general representation (12). The vortical component $\mathbf{v}_{\boldsymbol{\zeta}_{S}}$ is an extremely fast decaying function of distance from the actual position of the particle, while its potential correction $\nabla \Phi_{S}$ is not. In order to address the error propagation of the algorithm that will be illustrated in the next section, it is instrumental to explicitly provide an estimate on the order of magnitude of the field $\nabla \Phi_{S}$. The singular part of the pseudo-velocity is given by

$$
\mathbf{v}_{\boldsymbol{\zeta}_{S}}(\mathbf{x}, t)=\frac{1}{\rho_{f}} \int_{t-\epsilon_{R}}^{t^{+}} \mathbf{D}_{p}(\tau) g\left[\mathbf{x}-\mathbf{x}_{p}(\tau), t-\tau\right] d \tau,
$$

see eq. (13) for comparison. The equation for the potential correction is then

$$
\nabla^{2} \Phi_{S}=-\nabla \cdot \mathbf{v}_{\boldsymbol{\zeta}_{S}}=\frac{1}{\rho_{f}} \int_{t-\epsilon_{R}}^{t^{+}} \mathbf{D}_{p}(\tau) \cdot \nabla g\left[\mathbf{x}-\mathbf{x}_{p}(\tau), t-\tau\right] d \tau
$$

It follows

$$
\Phi_{S}=-\frac{1}{\rho_{f}} \int_{t-\epsilon_{R}}^{t^{+}} d \tau \mathbf{D}_{p}(\tau) \cdot \nabla \int_{\mathbb{R}^{3}} \frac{g\left[\mathbf{y}-\mathbf{x}_{p}(\tau), t-\tau\right]}{4 \pi|\mathbf{x}-\mathbf{y}|} d^{3} \mathbf{y},
$$

where $-1 /(4 \pi|\mathbf{x}-\mathbf{y}|)$ is the fundamental solution of the Laplace equation. From the solution (28) a rough estimate for the correction field $\nabla \Phi_{S}$ is 
immediately obtained as

$\left|\nabla \Phi_{S}\right| \leq \frac{1}{\rho_{f}} \sup _{t-\epsilon_{R} \leq \tau \leq t^{+}}\left|\mathbf{D}_{p}\right|\left|\nabla \otimes \nabla \int_{\mathbb{R}^{3}} \frac{\int_{t-\epsilon_{R}}^{t^{+}} g\left[\mathbf{y}-\mathbf{x}_{p}(\tau), t-\tau\right] d \tau}{4 \pi|\mathbf{x}-\mathbf{y}|} d^{3} \mathbf{y}\right|$

Sufficiently away from the particle, i.e. $\left|\mathbf{x}-\mathbf{x}_{p}\right| / d_{p} \gg 1$, the above estimate is asymptotically expressed as

$$
\left|\nabla \Phi_{S}\right| \leq \frac{1}{\rho_{f}} \int_{\mathbb{R}^{3}} \int_{t-\epsilon_{R}}^{t^{+}} g\left[\mathbf{y}-\mathbf{x}_{p}(\tau), t-\tau\right] d \tau d^{3} \mathbf{y} \sup _{t-\epsilon_{R} \leq \tau \leq t^{+}}\left|\mathbf{D}_{p}\right|\left|\nabla \otimes \nabla \frac{1}{4 \pi\left|\mathbf{x}-\mathbf{x}_{p}^{*}\right|}\right|,
$$

where $\mathbf{x}_{p}^{*}=\mathbf{x}_{p}\left(\tau^{*}\right), t-\epsilon_{R} \leq \tau^{*} \leq t^{+}$, is the position along the portion of the particle trajectory closest to the point $\mathbf{x}$. Given the known integral

$$
\int_{\mathbb{R}^{3}} \frac{1}{\left(2 \pi \sigma^{2}\right)^{3 / 2}} e^{-r^{2} /\left(2 \sigma^{2}\right)} d^{3} \mathbf{r}=1
$$

one ends up with

$$
\left|\nabla \Phi_{S}\right| \leq \sup _{t-\epsilon_{R} \leq \tau \leq t^{+}}\left|\mathbf{D}_{p}\right| \frac{\epsilon_{R}}{4 \pi \rho_{f}\left|\mathbf{x}-\mathbf{x}_{p}^{*}\right|^{3}},
$$

where the norm of the double tensor $\nabla \otimes \nabla\left[1 /\left(4 \pi \mid \mathbf{x}-\mathbf{x}_{p}^{*}\right)\right]$ is given by

$$
\left|\nabla \otimes \nabla \frac{1}{4 \pi\left|\mathbf{x}-\mathbf{x}_{p}^{*}\right|}\right|=\sup _{|\hat{\mathbf{e}}|=\mathbf{1}}\left[(\hat{\mathbf{e}} \cdot \nabla) \nabla \frac{1}{4 \pi\left|\mathbf{x}-\mathbf{x}_{p}^{*}\right|}\right]=\frac{1}{4 \pi\left|\mathbf{x}-\mathbf{x}_{p}^{*}\right|^{3}} .
$$

From the expression of the singular component of the pseudo-velocity 26 it is clear that, far from the particle, $\mathbf{v}_{\boldsymbol{\zeta}_{S}}$ decays exponentially fast, hence the far field dominating component of $\mathbf{v}_{S}$ is provided by the long range correction $\nabla \Phi_{S}$ that is order $\epsilon_{R} / r^{3}$. It is also clear that close to the particle the singular contribution is unbound. This singular near-field is however unessential as far as the relevant length scales of the system, either the smallest hydrodynamic scale $\eta$ or the inter-particle distance, are larger than $\sigma_{R}=\sqrt{2 \nu \epsilon_{R}}$. For this reason, it will be neglected when advancing the solution of one time step in the actual algorithm illustrated in the following sections. However, this highly localized field will eventually diffuse to larger scales at later times. Hence, the singular contribution that is neglected during a single time step is successively reintroduced in the field as soon as it 

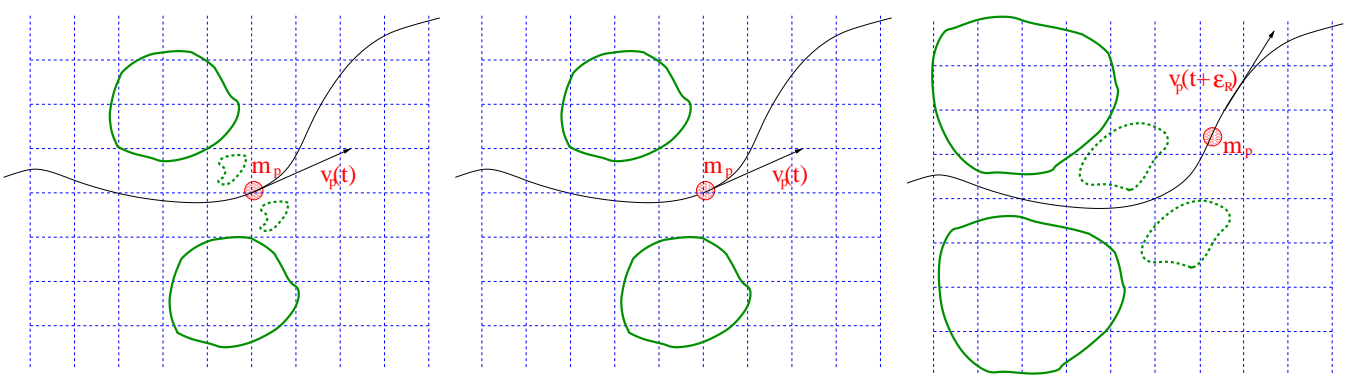

Figure 2: Coupling mechanism and regularization procedure. The green curves sketch the vorticity field. Left panel: the complete vorticity field generated by the particle at the current time $t$ is split into the regular $\boldsymbol{\zeta}_{R}(\mathbf{x}, t)$ (solid green line) and singular $\boldsymbol{\zeta}_{S}(\mathbf{x}, t)$ (dashed green line) components respectively. Central panel: only the regular component $\boldsymbol{\zeta}_{R}(\mathbf{x}, t)$ can be represented by the computational grid with mesh size $D x$ at the generic time $t$. Right panel: after the elapsed time $\epsilon_{R}$ (time $\left.t+\epsilon_{R}\right)$ the singular component of the vorticity field diffuses to scales large enough to be captured by the discrete grid. The momentum transfer towards the fluid occurs via viscous diffusion of the vorticity generated by the particle. When only the regularized field is considered a small error is incurred in the exchanged momentum. However, the successive diffusion of the singular field fully recovers the correct amount of vorticity at a successive time step. Thus the error does not accumulate in time and remains under control along the simulation.

reaches the smallest physically relevant scales of the system. This procedure guarantees that the error does not accumulate in time, thereby maintaining the accuracy of the calculation. Figure 2 sketches the decomposition into regular and singular fields, using the vorticity field to describe the process which is easier to visualize than the velocity field. The sketch highlight the singular production of vorticity by the particle, its diffusion, associated to momentum transfer the fluid, and the regularizing effects of viscosity. A crucial point is that the singular component of the field, which cannot be represented on a discrete mesh, is fully recovered at a successive time instant when its characteristic length-scale reaches the grid size. In the following section the convective effect of the singular field will be dealt with in more detail, to show that it is indeed negligible when the dynamics is observed at the relevant hydrodynamical scale. 


\subsection{Coupling with the carrier flow}

The regularized fluid velocity of the carrier flow in presence of the perturbing particles is obtained by aggregating the two contributions of the velocity decomposition $\mathbf{u}_{R}=\mathbf{w}+\mathbf{v}_{R}$ described in subsection $\$ 2.1$. The resulting field obeys the equations

$$
\begin{aligned}
& \nabla \cdot \mathbf{u}_{R}=0 \\
& \frac{\partial \mathbf{u}_{R}}{\partial t}+\mathbf{u}_{R} \cdot \nabla \mathbf{u}_{R}+\left\{\mathbf{v}_{S} \cdot \nabla \mathbf{u}_{R}+\mathbf{u}_{R} \cdot \nabla \mathbf{v}_{S}+\mathbf{v}_{S} \cdot \nabla \mathbf{v}_{S}\right\}= \\
& -\frac{1}{\rho_{f}} \nabla p+\nu \nabla^{2} \mathbf{u}_{R}-\frac{1}{\rho_{f}} \sum_{p}^{N_{p}} \mathbf{D}_{p}\left(t-\epsilon_{R}\right) g\left[\mathbf{x}-\mathbf{x}_{p}\left(t-\epsilon_{R}\right), \epsilon_{R}\right]
\end{aligned}
$$

with boundary and initial conditions given by

$$
\left.\mathbf{u}_{R}\right|_{\partial \mathcal{D}}=\mathbf{u}_{\text {wall }}-\left.\mathbf{v}_{S}\right|_{\partial \mathcal{D}}, \quad \mathbf{u}_{R}(\mathbf{x}, 0)=\mathbf{u}_{0}(\mathbf{x})
$$

where we have added the contributions arising from all the $N_{p}$ particles transported by the fluid. It should be stressed that the boundary condition for the regularized velocity $\mathbf{u}_{R}$ at $\partial \mathcal{D}$ needs taking the singular contribution $\mathbf{v}_{S}$ into account.

An interpretation of equation (34) could now be helpful. Along its motion the particle experiences the hydrodynamic force. In the formulation here proposed, the force is naturally regularized by viscous diffusion, hence the mollified Dirac delta functions takes the form of the fundamental solution of the diffusion equation. The effect of the hydrodynamic force is the generation of the regularized vorticity, (17), that is characterized by the smallest length-scale $\sigma_{R}=\sqrt{2 \nu \epsilon_{R}}$ where $\epsilon_{R}$ is the regularization diffusion time scale. A crucial point to be stressed again is that the hydrodynamic forcing acting on the regularized solution at time $t$ is the one experienced by the particles at a slightly previous time $t-\epsilon_{R}$ when their position were $\mathbf{x}_{p}\left(t-\epsilon_{R}\right)$. The net effect of the disperse phase on the regularized carrier flow field is then accounted for by the extra forcing term corresponding to the time-delayed hydrodynamic force expressed as the Gaussian $g\left[\mathbf{x}-\mathbf{x}_{p}\left(t-\epsilon_{R}\right), \epsilon_{R}\right]$ with variance $\sigma_{R}$.

The total field $\mathbf{u}$ will involve a singular part that is concentrated on scales smaller than the physically relevant ones. As such the singular contribution is actually neglected since only the regularized field needs to be considered. However contributions from the singular disturbance field $\mathbf{v}_{S}$ appear in the term in curly brackets in eq. (34). Indeed in the far field of the particles 
$\mathbf{v}_{S}$ was already shown to be of the order of $\epsilon_{R} / r^{3}$, which is negligible in comparison with the other terms in the equation. It is worth reminding that, at the successive time step, the corresponding contribution is re-introduced in the field, giving rise to no error accumulation in the long run. The crucial point here is that eq. (34) is taken to hold everywhere in $\mathcal{D}$, also close and inside the domains which are actually occupied by the particles. In this near field the curly bracket term needs to be treated with some care. In fact, due to the scale separation between $\mathbf{u}_{R}$ and $\mathbf{v}_{S}$, the filtering of the fields on a scale $\Delta$ which is order of the smallest hydrodynamic scale does not alter $\mathbf{u}_{R}$, i.e. denoting by $\hat{\mathbf{u}}_{R}$ the filtered field one has $\mathbf{u}_{R}=\hat{\mathbf{u}}_{R}$. In such conditions the equations for the regularized field follows by applying the filter to system (34). As a result of scale separation, the filter is actually acting only on the terms in curly brackets which involve the singular contribution $\mathbf{v}_{S}$. As explicitly shown in appendix A.4, a few detailed calculations show that the filtered terms give contribution of the order

$$
\begin{aligned}
& \widehat{\mathbf{v}_{S} \cdot \nabla \mathbf{v}_{S}} \sim R e_{p}\left(\frac{\sigma_{R}}{\Delta}\right)^{3} \frac{\mathbf{D}_{p}}{\rho_{f}} g_{\max } \\
& \widehat{\mathbf{v}_{S} \cdot \nabla \mathbf{u}_{R}} \sim \widehat{\mathbf{u}}_{R \cdot \nabla \mathbf{v}_{S}} \sim R e_{p}\left(\frac{\sigma_{R}}{\Delta}\right)^{2} \frac{\mathbf{D}_{p}}{\rho_{f}} g_{\max }
\end{aligned}
$$

where $g_{\max }=1 /\left(2 \pi \sigma_{R}^{2}\right)^{3 / 2}$ is the maximum of the mollified delta function. Clearly the above filtered convective terms are an order $R e_{p}$ smaller than the forcing term on the right hand side of eq. (34). Under the assumption of small particle Reynolds number they can be safely neglected in the evolution equation of the regularized field.

We like to stress the simplicity of the final equations that have to be solved,

$$
\begin{aligned}
& \nabla \cdot \mathbf{u}_{R}=0 \\
& \frac{\partial \mathbf{u}_{R}}{\partial t}+\mathbf{u}_{R} \cdot \nabla \mathbf{u}_{R}=-\frac{1}{\rho_{f}} \nabla p+\nu \nabla^{2} \mathbf{u}_{R}-\frac{1}{\rho_{f}} \sum_{p}^{N_{p}} \mathbf{D}_{p}\left(t-\epsilon_{R}\right) g\left[\mathbf{x}-\mathbf{x}_{p}\left(t-\epsilon_{R}\right), \epsilon_{R}\right] .
\end{aligned}
$$

The effects of the disperse phase on the carrier fluid is taken into account by an extra term in the Navier-Stokes equations. Under this point of view, any standard Navier-Stokes solver can be easily equipped with such extra term which is known in closed form. Furthermore each particle will produce an active forcing on the fluid localized in a sphere of radius order $\sigma_{R}$ centered 
at the particle position. In presence of many particles only the few grid points in the sphere of influence of the particle will receive the disturbance produced by the particle itself. Finally, the forcing term is grid independent in the sense that, once the grid spacing is refined ( $D x$ progressively getting smaller at fixed $\sigma_{R}$ ), any successively finer grid will only provide a better numerical approximation of the same forcing.

\subsection{Evaluation of the hydrodynamic force \& removal of self- interaction}

The dynamics of a point particle of mass $m_{p}$ in the relative motion with respect to a Newtonian fluid is described by the equation of motion,

$$
\frac{d \mathbf{x}_{p}}{d t}=\mathbf{v}_{p}(t), \quad m_{p} \frac{d \mathbf{v}_{p}}{d t}=\mathbf{D}_{p}(t)+\left(m_{p}-m_{f}\right) \mathbf{g},
$$

where $m_{f}$ is the displaced mass of fluid, $\mathbf{D}_{p}(t)$ is the hydrodynamic force, and g the acceleration due to gravity. Clearly, for the accurate evaluation of the particle trajectories and of the inter-phase momentum coupling, an accurate and efficient expression for the hydrodynamic force is mandatory. To obtain such expression one should reconsider the equation for the perturbation field $\mathbf{v}$ addressed in $\oint 2.1$.

As shown there, the perturbation due to the presence of a particle obeys the unsteady Stokes equation (4) where, noteworthy, the initial condition for the perturbation field $\mathbf{v}$ is homogeneous. Indeed, in our scheme, the solution of the unsteady Stokes equation for $\mathbf{v}$ at the generic time step provides the stress at the fluid-particle interface and ultimately yields the drag force. Luckily there is no need to work out the details, since [27] already provided the expression for the general unsteady drag force of a spherical particle when the field has homogenous initial condition, which is the case of interest here. Their solution can in fact be fully exploited to provide the drag force for the perturbation flow that is asymptotically expressed as (6) during the generic time step. 
Following [27] the force $\mathbf{D}_{p}(t)$ can be evaluated as

$$
\begin{aligned}
\mathbf{D}_{p}(t) & =6 \pi \mu a_{p}\left[\tilde{\mathbf{u}}\left(\mathbf{x}_{p}, t\right)+\frac{a_{p}^{2}}{6} \nabla^{2} \tilde{\mathbf{u}}\left(\mathbf{x}_{p}, t\right)-\mathbf{v}_{p}(t)\right] \\
& +\left.m_{f} \frac{D \tilde{\mathbf{u}}}{D t}\right|_{x_{p}}+\frac{1}{2} m_{f} \frac{d}{d t}\left[\tilde{\mathbf{u}}\left(\mathbf{x}_{p}, t\right)+\frac{a_{p}^{2}}{10} \nabla^{2} \tilde{\mathbf{u}}\left(\mathbf{x}_{p}, t\right)-\mathbf{v}_{p}(t)\right] \\
& +6 \pi \mu a_{p}^{2} \int_{0}^{t} d \tau \frac{1}{[\pi \nu(t-\tau)]^{1 / 2}} \frac{d}{d \tau}\left[\tilde{\mathbf{u}}\left(\mathbf{x}_{p}, \tau\right)+\frac{a_{p}^{2}}{6} \nabla^{2} \tilde{\mathbf{u}}\left(\mathbf{x}_{p}, \tau\right)-\mathbf{v}_{p}((3) p)\right]
\end{aligned}
$$

where $a_{p}=d_{p} / 2$ is the particle radius. Expression (39) involves the steady Stokes drag (first line), the added mass terms (second line), and the Basset history force (third line). In all terms the Faxen correction associated with spatial non-uniformity of the flow is included and, following the original derivation by [27, the velocity $\tilde{\mathbf{u}}\left(\mathbf{x}_{p}, t\right)$ must be interpreted as the fluid velocity, at the particle position, in absence of the particle self-interaction, i.e. $\tilde{\mathbf{u}}_{p}$ should account for the background - possibly turbulent - flow and for the disturbance generated by all the other particles except the $p$ th one, see also [5] and [14. In the regime of our interest, where the particle backreaction modifies the carrier flow, the correct calculation of $\tilde{\mathbf{u}}_{p}$ is crucial and calls for an effective procedure to deprive the field $\mathbf{u}(\mathbf{x}, t)$ evaluated at the particle position from the particle self-interaction contribution. Luckily, the (regularized) disturbance flow generated by each particle is known in closed form and can thus be easily removed from the complete field in computing the hydrodynamic force, at least for numerical algorithms using explicit time integration schemes. As an illustration, let us consider the simple case of heavy small particles, $\rho_{p} \gg \rho_{f}$, where the hydrodynamic force 39 reduces to the Stokes drag

$$
\mathbf{D}_{p}(t)=6 \pi \mu a_{p}\left[\tilde{\mathbf{u}}\left(\mathbf{x}_{p}, t\right)-\mathbf{v}_{p}(t)\right]
$$

The explicit calculation of the velocity $\mathbf{v}_{R}\left(\mathbf{x}-\mathbf{x}_{0}, t\right)$ induced at time $t$ and position $\mathbf{x}$ by a particle located at $\mathbf{x}_{0}$ is provided in appendix A.5. This result can be exploited to remove the self-interaction term in the illustrative case of an explicit Euler time advancement algorithm. Indeed, in this case, to correctly evaluate the right hand side of equation (40) it suffices to subtract from $\mathbf{u}\left(\mathbf{x}_{p}, t\right)$ the value $\mathbf{v}_{R}\left[\mathbf{x}_{p}(t)-\mathbf{x}_{p}(t-D t), D t\right]$ induced at time $t$ at the current particle position $\mathbf{x}_{p}(t)$ by the same particle when it was placed at $\mathbf{x}_{p}(t-D t)$. The same kind of reasoning can be straightforwardly extended to other explicit time integration schemes, e.g. to each intermediate step of 
a Runke-Kutta algorithm and to the different contributions in the general expression of the force (39), e.g. bubbly flows [8].

Before closing this section devoted to force evaluation, a final note is in order concerning the Basset force: it represents the effects on the force due to the particle-fluid interaction during the particle previous motion before the actual time $t$. In cases where the particle do not modify the carrier flow, see the derivation by [27], this interaction is modeled by a memory convolution integral which mimics the particle vorticity production and its viscous diffusion occurring from the initial time $t=0$ up to the actual time $t$. In our case, the carrier fluid is perturbed step by step by the particle motion (two-way coupling regime) and the diffusion of the vorticity produced by the particle during the past motion before the actual time $t$ is captured without any modeling by equations (37). Hence, the time integral must model the only vorticity production occurring during the last time step $D t$, i.e. the memory integral is limited to a single time step of the eventual integration algorithm. Actually, the effects of the previous history come in through the boundary condition of equations (4) where the field $\mathbf{w}$ must be interpret as the background velocity acting on the particle, i.e. as the carrier flow velocity field that would occur at the particle boundary during the last time step in absence of the particle. For small particles such field reduces to the value at particle center plus a Faxen-like correction accounting for spatial flow variations on the scale of the particle.

\section{Algorithm validation}

The methodology illustrated in the previous sections needs to be validated. We will address several test cases where analytical data can be employed for comparison. To better focus our attention on the interaction between the fluid and the disperse phase, we will consider a periodic box $\mathcal{D}$ free from solid boundaries which may hinder the analysis. The numerical solution of equation (25) and (34) for the carrier fluid is based on a pseudospectral Fourier-based spatial discretization where the non linear terms are calculated by the standard $3 / 2$ dealiasing procedure. Time advancement is achieved by a low-storage semi-implicit Runge-Kutta method with a fourthorder Adams-Bashforth formulation for the convective terms and an implicit Crank-Nicholson formula for the diffusive terms. The details on the implementation are described elsewhere, see [15]. 


\subsection{Response to a localized force}

We start by addressing a simple case where a known small amplitude constant force $\mathbf{F}_{0}$ is applied at a fixed point $\mathbf{x}_{p}$ to the fluid which is initially at rest in the domain $\mathcal{D}$. Due to the small amplitude of the forcing, the flow is assumed to obey the linear, unsteady Stokes equations. Equation (25) is suitable of an analytical solution which, in terms of vorticity, is provided by equation (17). This reference solution allows to verify that the algorithm correctly transfers the proper impulse to the fluid, a crucial aspect in view of simulations in the two-way coupling regime.

Due to periodicity, the exact impulse, $\mathbf{I}_{E}=\mathbf{F}_{0} t=\int_{\mathcal{D}} \rho_{f} \mathbf{u}(\mathbf{x}, t) d^{3} \mathbf{x}$, can be expressed in terms of the vorticity moment ([39]),

$$
\mathbf{I}_{E}(t)=\frac{1}{2} \rho_{f} \int_{\Omega} \mathbf{x} \times \boldsymbol{\zeta}(\mathbf{x}, t) d^{3} \mathbf{x} .
$$

The error $E_{I}=\left|\mathbf{I}_{E}(t)-\mathbf{I}_{N}(t)\right|$, where $\mathbf{I}_{N}(t)$ is the estimate of (41) from the numerical solution, is shown in semi-logarithmic scale in the left panel of figure 3 as a function of the normalized time $t / \epsilon_{R}$ for a fixed value of the regularization timescale $\epsilon_{R}=0.01$ and different spatial resolutions, namely the ratio $\sigma_{R} / D x$. In the unresolved cases $\left(\sigma_{R} / D x<1\right)$, the error is order one and increases in time. In contrast, when a proper spatial resolution is adopted, i.e. $\sigma_{R} / D x>1$, the error becomes progressively smaller as the resolution is increased and stays constant in time. In other words, as the simulation advances in time $E_{I}$ does not accumulate. The right panel of figure 3 reports the supreme $\sup _{t \geq 0}\left|\mathbf{I}_{E}(t)-\mathbf{I}_{N}(t)\right|$ as a function of the ratio $\sigma_{R} / D x$. This plot emphasized the convergence rate of the impulse against the spatial resolution at a fixed value of $\epsilon_{R}$. The inset shows $\sup _{t \geq 0} E_{I}$ in a different manner. Here the spatial resolution is fixed, $\sigma_{R} / D x=1$, and the regularization timescale is progressively reduced denoting convergence also with respect to the parameter $\epsilon_{R}$.

The impulse, though a fundamental quantity, does not retain any information concerning the spatial structure of the fluid field. To go more in depth into the convergence analysis we have addressed the vorticity field. The error is now defined by using the standard $L^{2}$ norm as $E_{\boldsymbol{\zeta}}=\left\|\boldsymbol{\zeta}_{E}-\boldsymbol{\zeta}_{N}\right\|_{2}$ where the subscripts refer to the exact regularized solution (17) and its nu-

merical counterpart. The error $E_{\zeta}$ is shown in the left panel of figure 4 as a function of time for $\epsilon_{R}=0.01$ and several values of the ratio $\sigma_{R} / D x$. When a proper spatial resolution is adopted, also the error $E_{\zeta}$ stays constant in time or decreases. Note that the largest error is achieved at the early stages of the simulation when the force is applied to the fluid at rest. 

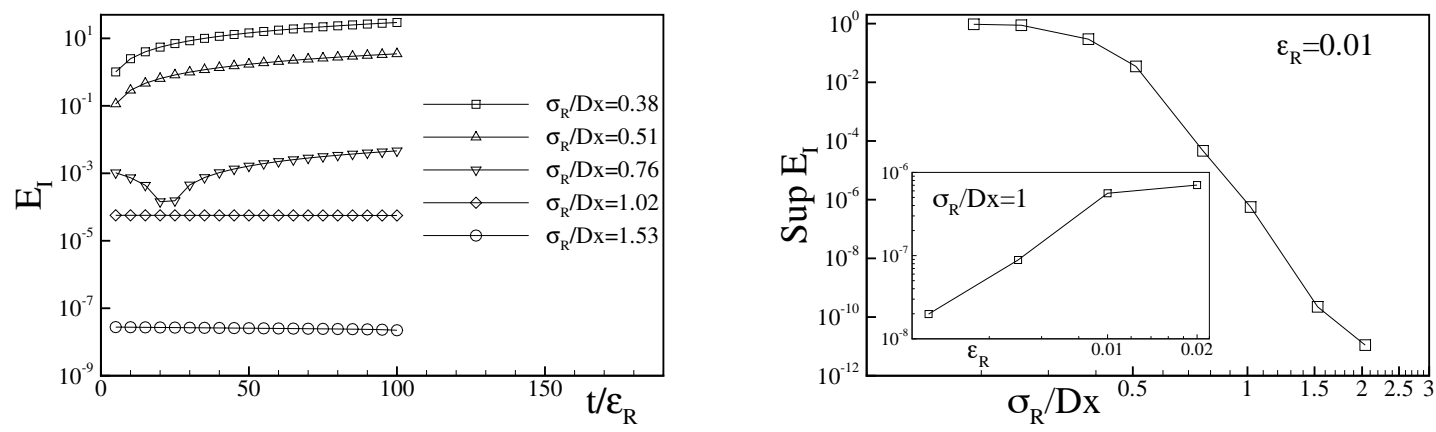

Figure 3: Convergence study in the case of a constant force $\mathbf{F}_{0}=(1,0,0)$ applied in a fixed point $\mathbf{x}_{p}$ to the fluid initially at rest in a periodic box $\mathcal{D}$. Left: the error $E_{I}$ of the total impulse, see text for definition, is plotted against the normalized time $t / \epsilon_{R}$ for a fixed regularization timescale $\epsilon_{R}=0.01$ and different values of the spatial resolution $\sigma_{R} / D x$. Right: the supreme $\sup _{t>0} E_{I}$ is plotted against the ratio $\sigma_{R} / D x$ for $\sigma_{R}=0.01$. The inset reports the supreme $\sup _{t \geq 0} E_{I}$ for different values of $\epsilon_{R}$ and a fixed spatial resolution $\sigma_{R} / D x=1$.

In any case, the supreme $\sup _{t \geq 0} E_{\zeta}$ converges with respect to the refinement of the spatial resolution as shown in the right panel of the figure. The inset reports $\sup _{t \geq 0} E_{\zeta}$ against the regularization timescale $\epsilon_{R}$ for a fixed spatial resolution documenting the convergence of $\sup _{t \geq 0} E_{\zeta}$ also with respect to $\epsilon_{R}$.

A more detailed insight concerning the ensuing fluid motion generated by the fixed force is achieved by a direct inspection of the flow field. Figure 5 reports the fluid velocity in a one dimensional cut across the complete three-dimensional field. It is useful to fix the notation: the constant force has cartesian components $\mathbf{F}_{0}=\left(F_{x}, F_{y}, F_{z}\right)$, the corresponding velocity vector is $\mathbf{u}=(u, v, w)$ and the distance from $\mathbf{x}_{p}$ is measured by the vector $\mathbf{r}=\mathbf{x}-\mathbf{x}_{p}$ whose cartesian components are $\mathbf{r}=\left(r_{x}, r_{y}, r_{z}\right)$. In the plots of figure 5 the force is $\mathbf{F}_{0}=(1,0,0)$. The figure presents the velocity disturbance $u$ in the direction of the force as a function of the separation $r_{x}$ along a one dimensional cut aligned with the x-axis passing through the point where the force is applied. The different profiles pertain to simulations which share the same regularization timescale $\epsilon_{R}=0.01$ but differ for the spatial resolution. Namely, a typical unresolved case $\sigma_{R} / D x=0.38$ and a resolved simulation $\sigma_{R} / D x=1$ are compared against the exact solution at two different times 

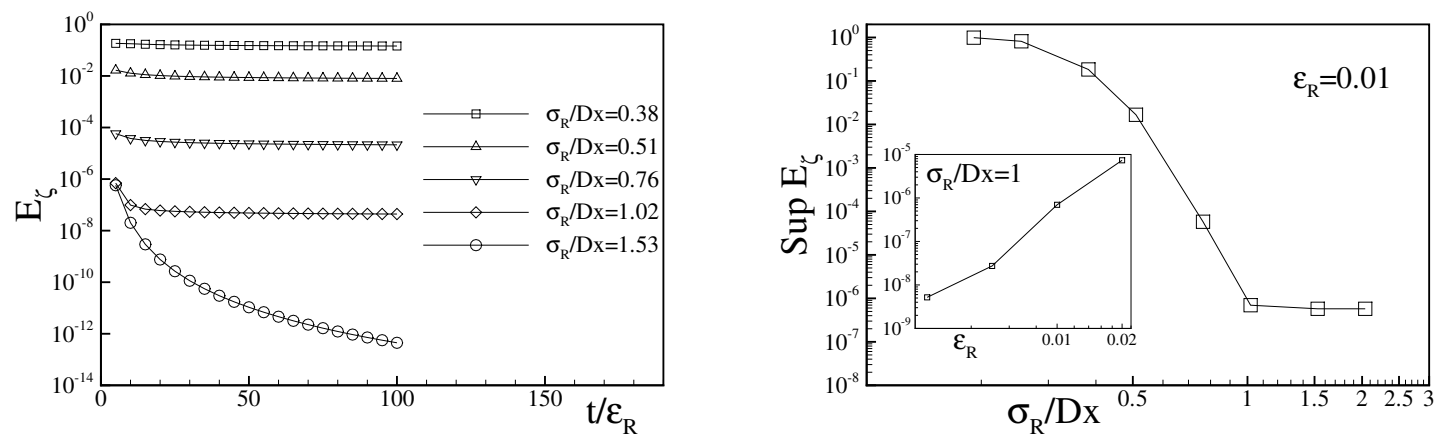

Figure 4: Convergence study in the case of a constant force $\mathbf{F}_{0}=(1,0,0)$ applied in a fixed point $\mathbf{x}_{p}$ to the fluid initially at rest in a periodic box $\mathcal{D}$. Left: the error $E_{\boldsymbol{\zeta}}$, see text for definition, is plotted against the normalized time $t / \epsilon_{R}$ for a fixed regularization timescale $\epsilon_{R}=0.01$ and different values of the spatial resolution $\sigma_{R} / D x$. Right: the supreme $\sup _{t \geq 0} E_{\zeta}$ is shown as a function of the ratio $\sigma_{R} / D x$ for $\sigma_{R}=0.01$. The inset reports the supreme $\sup _{t \geq 0} E_{\zeta}$ versus the regularization timescale $\epsilon_{R}$ for a fixed spatial resolution $\sigma_{R} / D x=1$.

along the simulation. In fact, when a fixed constant force is applied to the fluid, an exact solution can be easily determined in closed form by evaluating the time convolution integral between the unsteady Green tensor, see appendix equation (60), and the force $\mathbf{F}_{0}$. After some algebra, the fluid velocity disturbance in the direction of the force reads

$$
u(\mathbf{r}, t)=\frac{1}{4 \pi \mu r}\left[\frac{1}{2 \eta_{t}^{2}} \operatorname{erf}\left(\eta_{t}\right)-\operatorname{erf}\left(\eta_{t}\right)-\frac{1}{\sqrt{\pi} \eta_{t}} \exp \left(-\eta_{t}^{2}\right)+1\right]
$$

where $\eta_{t}=r / \sqrt{4 \nu t}$ and $r=\sqrt{r_{k} r_{k}}$. As shown in the figure 5, when $\sigma_{R} / D x \geq 1$ the present algorithm well reproduces the exact solution. Note that insufficient spatial resolution results in a clear underestimate of the fluid velocity disturbance. This is emphasized by the plots reported in the insets figure 5 where the data are represented in a semi-logarithmic scale.

Figure 6 documents the behavior of the ERPP method when the spatial resolution $\sigma_{R} / D x$ is kept fixed and the regularization timescale is progressively reduced. In fact, as $\epsilon_{R}$ is decreased, the numerical solution describes a progressively wider range of the exact solution avoiding in all cases the occurrence of the singularity at the point $\mathbf{x}_{p}$ where the force is applied, $r_{x}=0$ in the plot. The different cases share the same far field behavior away from 

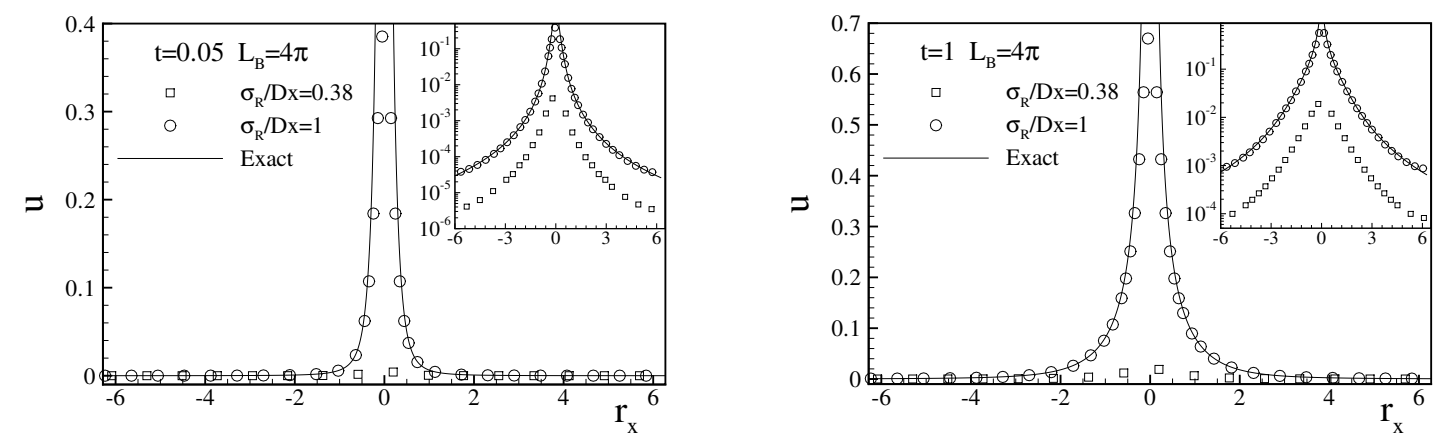

Figure 5: Fluid velocity disturbance generated by a fixed constant force $\mathbf{F}_{0}=(1,0,0)$ on an initially motionless fluid contained in a periodic box $L_{B}=4 \pi$. The $1 D$ profile representing the fluid velocity in the direction of the force (symbols) is compared against the exact solution (solid line). The velocity component $u(\mathbf{r}, t)$ in the $x$-direction is plotted against the separation $r_{x}$ for $\epsilon_{R}=0.01$ and two spatial resolutions, namely $\sigma_{R} / D x=$ $0.38(\square)$ and $\sigma_{R} / D x=1(\bigcirc)$. Left: velocity disturbance at $t=0.05$. Right: velocity disturbance at $t=1$. In the insets of the two panels the data are plotted in a semi-logarithmic scale. 

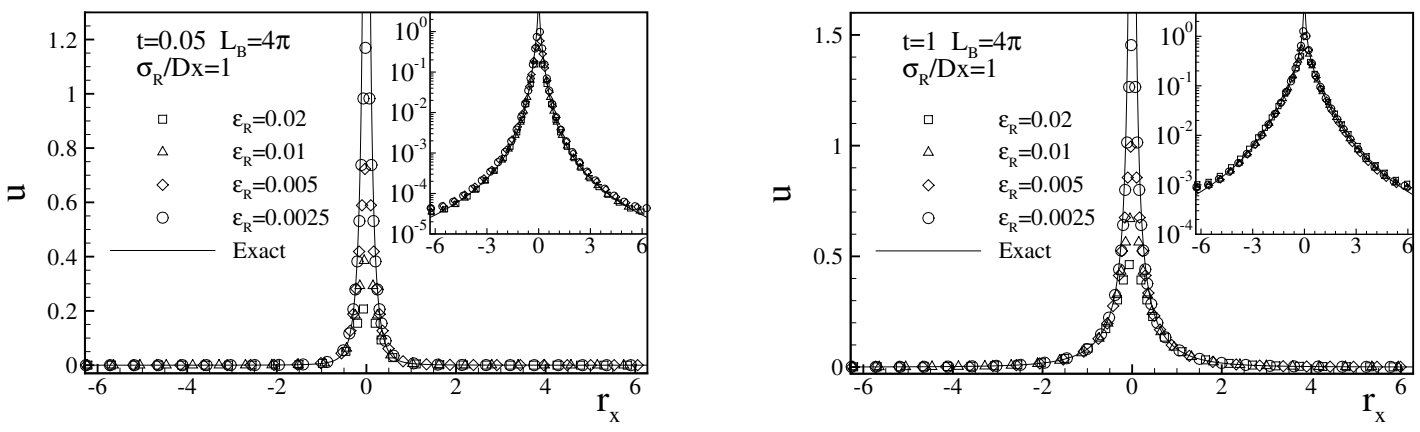

Figure 6: Fluid velocity disturbance generated by a fixed constant force $\mathbf{F}_{0}=(1,0,0)$ on an initially motionless fluid contained in a periodic box $L_{B}=4 \pi$. The $1 D$ profile representing the fluid velocity in the direction of the force (symbols) is compared against the exact solution (solid line). The velocity component $u(\mathbf{r}, t)$ in the $x$-direction is plotted against the separation $r_{x}$ for several values of the regularization timescale, $\epsilon_{R}=0.01$ $(\square) ; \epsilon_{R}=0.02(\triangle) ; \epsilon_{R}=0.005(\diamond) ; \epsilon_{R}=0.0025(\bigcirc)$, at a fixed spatial resolution $\sigma_{R} / D x=1$. Left: velocity disturbance at $t=0.05$. Right: velocity disturbance at $t=1$. The insets of the two panels show the data plotted in a semi-logarithmic scale.

$\mathbf{x}_{p}$ irrespective of the value of $\sigma_{R}$ as emphasized by the plots in the insets of figure 6 where the velocity disturbance is represented in a semi-logarithmic scale. In summary, the solution provided by the ERPP retains the relevant features of the exact solution and avoids the occurrence of the singularity at $\mathbf{x}_{p}$ which is clearly an unwanted trait in any numerical solution.

Figure 7 reinforces the conclusion of the previous analysis by showing the fluid velocity component in the direction of the force $u\left(r_{y}\right)$ as a function of the distance $r_{y}$ in a transversal one-dimensional cut through the point of application of the force. Finally figure 8 reports the fluid velocity component in the direction of the force plotted against the normalized distance $r_{x} / \sigma_{R}$ and $r_{y} / \sigma_{R}$. The discussion of these plots requires some care. The ERPP model has been conceived to describe the far field effect produced on the fluid by a point-like particle avoiding the occurrence of singularities in the point $\mathbf{x}_{p}$ where the particle is located. In fact, the disturbance produced by the particle is described by retaining only the first term in the multipole expansion of the general solution of the unsteady Stokes flow and the regularization timescale $\epsilon_{R}$ accounts for the viscous diffusion process 

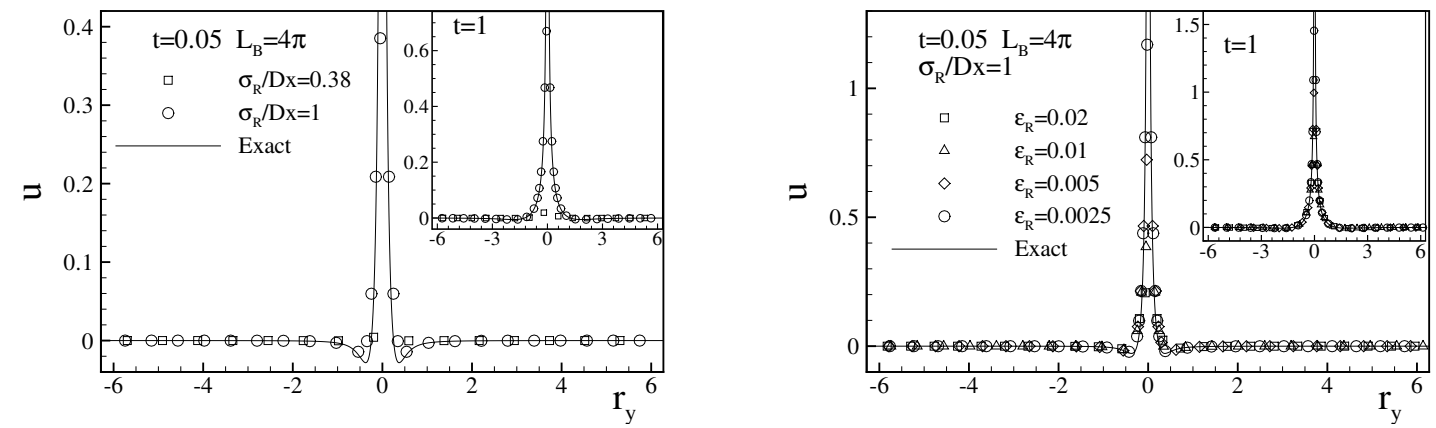

Figure 7: Fluid velocity disturbance generated by a fixed constant force $\mathbf{F}_{0}=(1,0,0)$ on an initially motionless fluid contained in a periodic box $L_{B}=4 \pi$. The $1 D$ profile representing the fluid velocity component $u(\mathbf{r}, t)$ in the $x$-direction (symbols) is plotted against the separation $r_{y}$. The exact solution (solid line) is reported for comparison. Left: fluid velocity disturbance at $t=0.05$ (main panel) and $t=1$ (inset) plotted for a fixed value of $\epsilon_{R}=0.01$ and two spatial resolutions namely $\sigma_{R} / D x=0.38$ and $\sigma_{R} / D x=1$. Right: fluid velocity disturbance at $t=0.05$ (main panel) and $t=1$ (inset) plotted for several values of $\epsilon_{R}$ and a fixed spatial resolution $\sigma_{R} / D x=1$. 

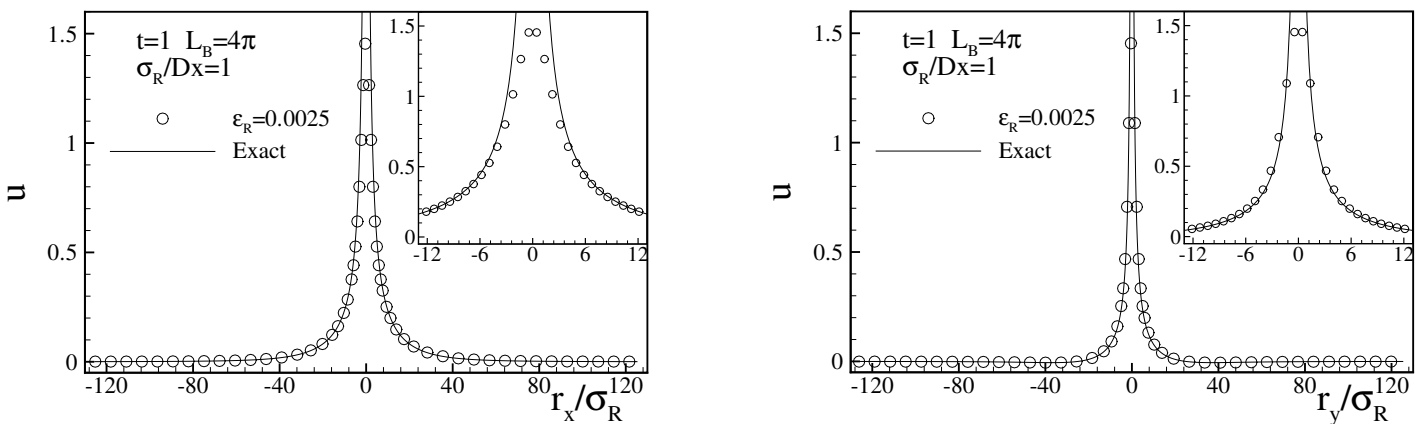

Figure 8: Fluid velocity disturbance generated by a fixed constant force $\mathbf{F}_{0}=(1,0,0)$ on an initially motionless fluid contained in a periodic box $L_{B}=4 \pi$. The velocity component $u(\mathbf{r}, t)$ in the $x$-direction (direction of the force, symbols) is plotted against the normalized separation $r_{x} / \sigma_{R}$ (left panel) and $r_{y} / \sigma_{R}$ (right panel) for $\epsilon_{R}=0.0025$ and $\sigma_{R} / D x=1$. The inset of the two panels provides a close-up view of the solution at $\mathbf{x}_{p}=0$.

which naturally regularizes the solution. Hence, the solution provided by the ERPP has un intrinsic inner cut-off provided by $\sigma_{R}$ and is expected to reproduce the disturbance flow generated by a point-particle in the far field. This is indeed what happens and what is documented by the plots in figure 8. The regularized solution stays on top of the exact solution everywhere, see e.g. the main panels of figure 8 which, on the scale of the complete computational domain $\mathcal{D}$, reports the fluid velocity disturbance produced by the particle. The insets of figure 8 show the same data in proximity of the origin where the particle is placed. Such representation emphasizes that, after a distance of a few $\sigma_{R}$, the ERPP solution falls on top of the exact solution. The threshold $3 \sigma_{R}$ can be safely assumed as an inner cut-off for the disturbance flow field produced by the particle.

\subsection{Unsteady motion of an isolated particle}

The following subsections address more realistic cases where the point $\mathbf{x}_{p}$ is allowed to move according to equations (38) with the initial conditions $\mathbf{x}_{p}(t=0)=\mathbf{x}_{p}^{0} ; \mathbf{v}_{p}(t=0)=0$. In order to proceed gradually we will first discuss a series of tests where the particle motion is not affected by the fluid velocity disturbance that the particle generates. In such decoupled cases the fluid disturbance field is still amenable of exact solutions which 
can be employed for further comparisons. Successively, we will consider the fully coupled case where the dynamics of the particles and of the fluid are intertwined.

\subsubsection{Imposed particle motion}

We consider the motion of a small particle subjected to an external force, e.g. gravity, and to the Stokes drag. The particle velocity is given by the solution of the equation

$$
m_{p} \frac{d \mathbf{v}_{p}}{d t}=m_{p} \mathbf{g}-6 \pi \mu a_{p} \mathbf{v}_{p}(t)
$$

namely

$$
v_{p}(t)=v_{t}\left(1-e^{-t / \tau_{p}}\right)
$$

where $v_{p}(t)$ denotes the particle velocity in the direction of the gravity acceleration, say the $x$-direction, $v_{t}=\tau_{p} g$ is the particle terminal velocity and $\tau_{p}=\rho_{p} d_{p}^{2} / 18 \mu$ the Stokes relaxation timescale. In this framework the motion of the particle is imposed and its dynamics is decoupled from the dynamics of the carrier fluid.

The left panel of figure 9 reports the fluid velocity disturbance produced by the moving particle at $t / \tau_{p}=20$ when the particle has reached its terminal velocity $v_{t}$. The fluid velocity profile is plotted for two cases which differs for the value of the regularization timescale $\epsilon_{R}$. Once again the value of $\epsilon_{R}$ controls the regularized near field and does not affect the far field, see the inset of the figure. In this case the fluid velocity has an explicit solution given by the time convolution integral of the unsteady Stokeslet, equation (60), and the the hydrodynamic force $D_{p}(t)$ where the Stokeslet is placed at the instantaneous position of the particle evolving as specified by equation (44). Performing the time convolution integral is now a little more tricky than in the previous example since $\mathbf{r}=\mathbf{x}-\mathbf{x}_{p}(t)$ where $\dot{x}_{p}=v_{p}(t)$. The integration of the expression

$$
u_{i}(\mathbf{x}, t)=\int_{0}^{t} G_{i k}\left[\mathbf{x}-\mathbf{x}_{p}(\tau), t-\tau\right] D_{k}^{p}(\tau) \quad d \tau
$$

in a closed form becomes cumbersome even though the integral can be evaluated numerically by a quadrature formula. Indeed, such numerical approximation of the exact solution can be still used for useful comparisons. The data produced by the ERPP algorithm are compared with this reference solution in figure 9. The plots show that the ERPP approach is able to 
capture the expected solution and provides a consistent regularization of the singularity which occurs at $\mathbf{x}_{p}$. The large fluid domain, note that the box size is $L_{B}=4 \pi$, allows a good approximation of the unbounded domain where the reference solution (45) holds. The right panel of figure 9 provides the fluid velocity disturbance produced by particles with different terminal velocities $v_{t}$. The effect of increasing the terminal velocity is worth discussing. As $v_{t}$ is increased the front-aft symmetry in the disturbance flow is progressively broken. This symmetry breaking is indeed easily explained in terms of vorticity released along the path of the moving particle. In fact in the body-fixed frame, the convection term $\mathbf{v}_{p} \cdot \nabla \mathbf{u}$ is responsible of the constant velocity advection of the vorticity even in a Stokes regime. In order to capture the tails of the disturbance flow in the far field and to avoid confinement effects introduced by the periodic boundary conditions, the computational box is now $L_{B}=8 \pi$ wide.

We conclude the discussion by presenting in figure 10 the comparison between the ERPP solution and what one would obtain by using the classical Particle In Cell (PIC) approach. As expected, the solution provided by the PIC method is grid dependent, as demonstrated by comparing the disturbance velocity profile of two simulations which share the same physical parameters but differ for the grid resolution, namely $N=192^{3}$ and $N=384^{3}$ Fourier modes. As the grid is refined a singular-like behavior occurs at $\mathbf{x}_{p}$ and the field is characterized by numerical aliasing, see e.g. the top right inset where the velocity profile is plotted in a semi-logarithmic scale. In contrast, once the regularization timescale $\epsilon_{R}$ is fixed, the ERPP approach provides a numerically convergent, asymptotically grid-independent solution. This behavior can be better appreciated in the top left inset where a close-up view of the velocity disturbance is reported. In a nutshell the ERPP retains all the features of the physical solution produced by a small point-like particle except for the (undesired) singularity which unavoidably occurs at $\mathbf{x}_{p}$. The regularization of the solution is controlled by the timescale $\epsilon_{R}$ which is related to a diffusive lengthscale $\sigma_{R}$. Indeed $\sigma_{R}$ is naturally introduced by the process of vorticity diffusion and can be fixed on a physical ground. For instance, in a turbulent flow, velocity fluctuations are physically irrelevant below the Kolmogorov lengthscale $\eta$. At the same time the effects that a swarm of point-like particles generates on length scales larger than $\eta$ is physically relevant. In such framework the regularization lengthscale $\sigma_{R}$ is naturally selected as $\sigma_{R}=\eta$. 

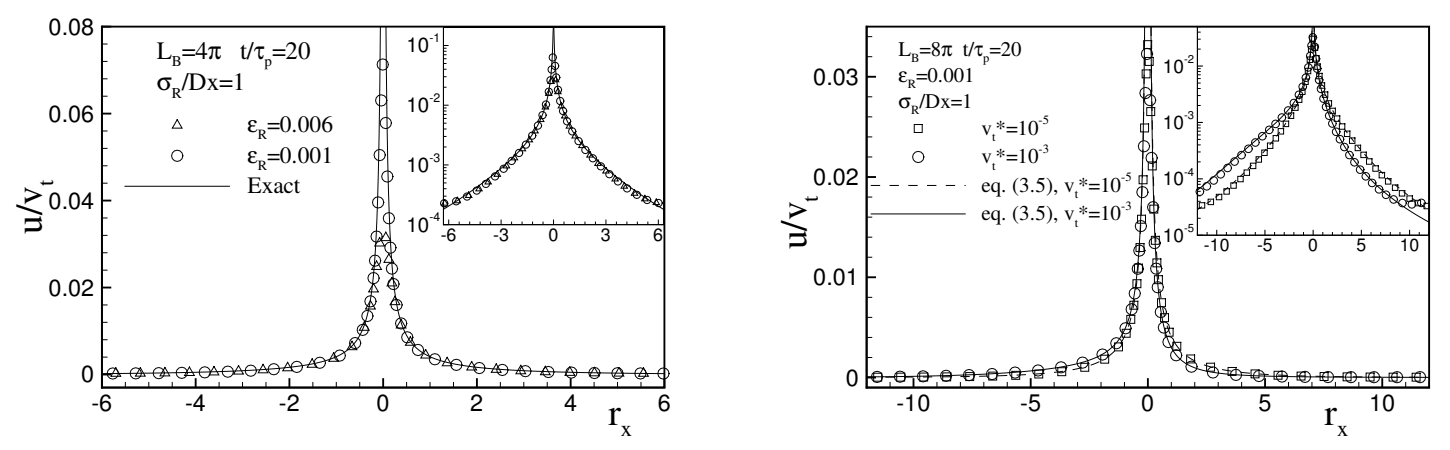

Figure 9: Normalized velocity disturbance produced by a particle moving with velocity $v_{p}(t)=v_{t}\left(1-e^{-t / \tau_{p}}\right)$ in the $x$-direction in a periodic box $L_{B}=4 \pi$. The normalized velocity disturbance $u / v_{t}$ in the direction of the particle motion, is plotted against the separation $r_{x}$ at time $t / \tau_{p}=20$ when the particle has reached the terminal velocity $v_{t}$. Left panel: data obtained for different values of the regularization timescale $\epsilon_{R}=0.006(\triangle)$; $\epsilon_{R}=0.001(\bigcirc)$ at a fixed spatial resolution $\sigma_{R} / D x=1$, are compared against the exact solution (solid line) given by equation (45). The inset reports the data of the main panel plotted in a semi-logarithmic scale. Right panel: data pertaining particles with with different terminal velocities, $v_{t}^{*}=$ $v_{t} d_{p} / \nu=10^{-5}(\square) ; v_{t}^{*}=10^{-3}(\triangle)$, are compared against the corresponding exact solution (45), dashed and solid line respectively, in a periodic box $L_{B}=8 \pi$. The inset reports the date of the main panel plotted in a semilogarithmic scale. 


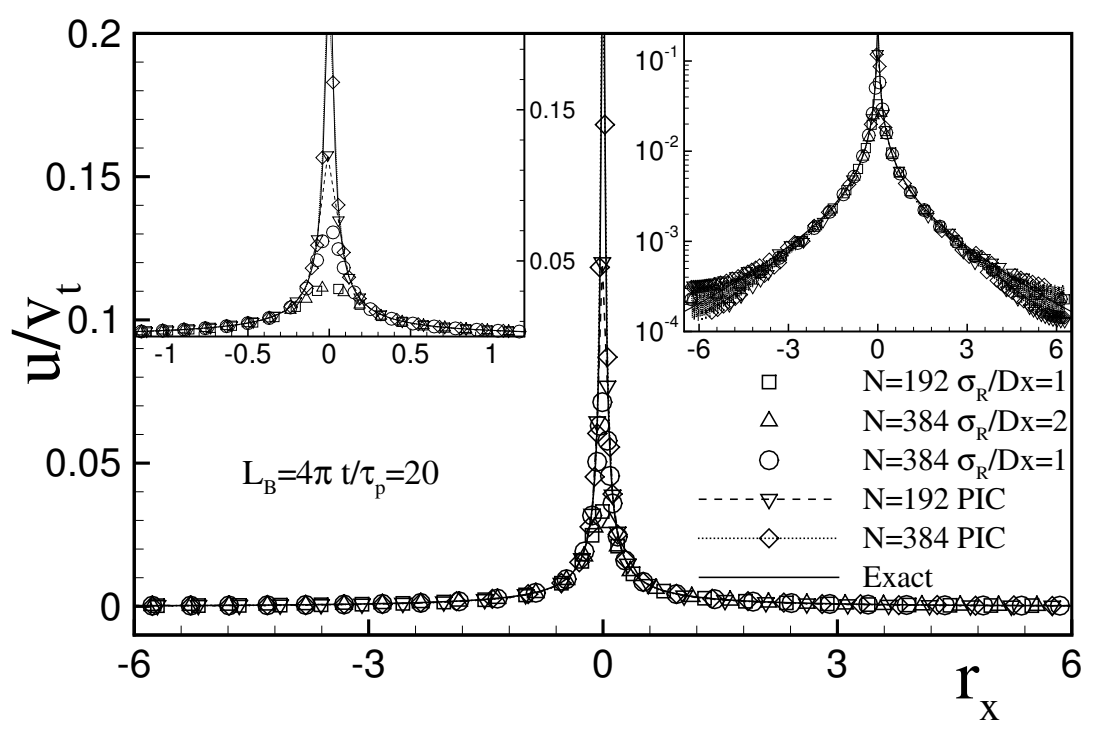

Figure 10: Normalized velocity disturbance at $t / \tau_{p}=20$ produced by a particle moving with velocity $v_{p}(t)=v_{t}\left(1-e^{-t / \tau_{p}}\right)$ in the $x$-direction in a periodic box $L_{B}=4 \pi$. The velocity profile calculated with the ERPP for different values of the regularization timescale $\epsilon_{R}=0.006, N=192^{3}$ Fourier modes, $\sigma_{R} / D x=1(\square) ; \epsilon_{R}=0.006, N=384^{3}$ Fourier modes, $\sigma_{R} / D x=2,(\triangle) ; \epsilon_{R}=0.001, N=384^{3}$ Fourier modes, $\sigma_{R} / D x=1$, $(\bigcirc)$; is compared against the exact solution (solid line) and corresponding results obtained by the PIC approach, $N=192^{3}$ Fourier modes, $(\nabla) ; N=384^{3}$ Fourier modes, $(\diamond)$. Top left panel: close-up view of the velocity disturbance near the singular point $\mathbf{x}_{p}$. Top right inset: velocity profile plotted in semilogarithmic scale. 


\subsubsection{Particle motion in the coupled regime}

This subsection addresses the unsteady motion of a particle which settles from rest under the action of gravity in the coupled regime where the particle induces a disturbance in the surrounding fluid and such disturbance enters the expression of the hydrodynamic force. For simplicity we will consider small particles much heavier than the surrounding fluid, i.e. $\rho_{p} \gg \rho_{f}$, where the only relevant force is the Stokes drag. The general expression of the force 39 simplifies to

$$
\mathbf{D}_{p}(t)=m_{p} \mathbf{g}+6 \pi \mu a_{p}\left[\tilde{\mathbf{u}}\left(\mathbf{x}_{p}, t\right)-\mathbf{v}_{p}(t)\right] .
$$

Following the discussion of section 2.5 the velocity $\tilde{\mathbf{u}}\left(\mathbf{x}_{p}, t\right)$ must be interpreted as the background fluid velocity in absence of the pth particle, e.g. turbulent fluctuations plus the disturbance flow generated by all the other particles. This makes the calculation of the hydrodynamic force particularly challenging in the two-way coupling regime. In the particular case where only one particle is considered the value $\tilde{\mathbf{u}}\left(\mathbf{x}_{p}, t\right)$ should be set to zero. However this way of proceeding is unfeasible in the general case where many particles are present since the value of $\tilde{\mathbf{u}}\left(\mathbf{x}_{p}, t\right)$ must also account for the velocity disturbance generated by all the other particles and the background flow. This conundrum can be disentangled in the context of the ERPP approach since the disturbance flow produced by the $p$ th particle on itself is known in a closed form and thus can be removed from the background fluid velocity $\mathbf{u}\left(\mathbf{x}_{p}, t\right)$ even in presence of many other particles.

The two panels of figure 11 provide evidence to the above considerations. The plots report the particle velocity normalized with the settling velocity $v_{t}$ as a function of the dimensionless time $t / \tau_{p}$ both for the ERPP calculation and for the PIC approach. The particle trajectory should be compared with the reference solution given by equation (44). The left panel shows the particle velocity calculated by the ERPP method for different values of the ratio $d_{p} / \sigma_{R}$. We recall that our approach is designed to model the disturbance flow produced by point-like particles, i.e. particles whose diameter $d_{p}$ is much smaller that any other lengthscale in the system. In the ERPP approach, in absence of any other length-scales introduced by the background flow, the only significant lengthscale is the diffusive scale $\sigma_{R}$. Hence, the nominal diameter of the particle should be smaller than $\sigma_{R}$. Indeed, as the ratio $d_{p} / \sigma_{R}$ decreases the particle velocity rapidly approaches the reference curve provided by equation (44). When the scale $\sigma_{R}$ has been fixed the ERPP gives a grid-independent solution as can be appreciated in figure 11 where two trajectories which share the same $\sigma_{R}$ but have different 

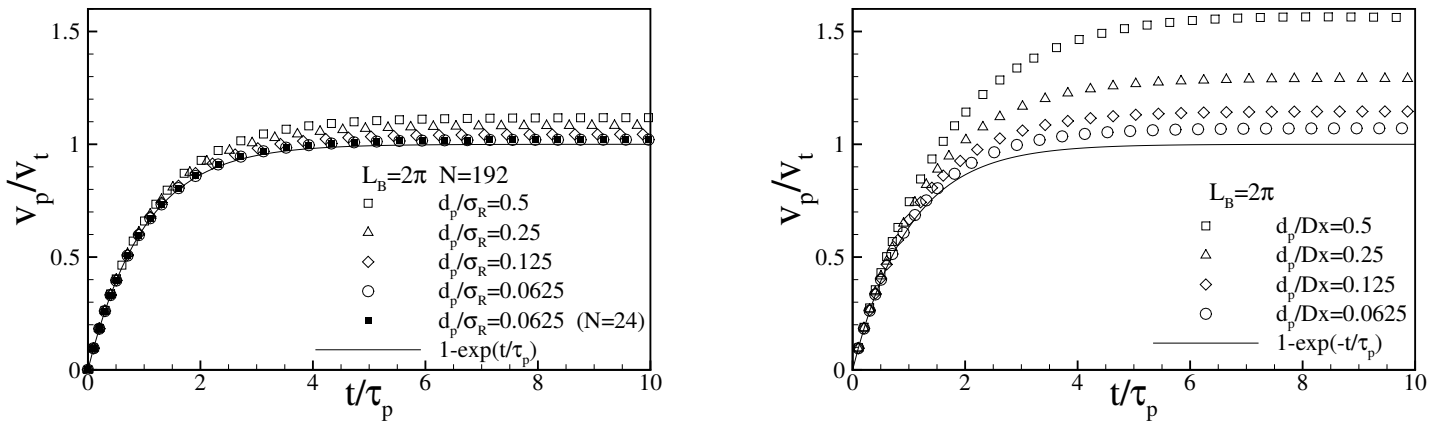

Figure 11: Normalized particle velocity $v_{p} / v_{t}$ as a function of the dimensionless time $t / \tau_{p}$. Left panel: particle velocity in the ERPP simulations for different values of the ratio; $d_{p} / \sigma_{R}=0.5,(\square) ; d_{p} / \sigma_{R}=0.25,(\triangle)$; $d_{p} / \sigma_{R}=0.125,(\diamond) ; d_{p} / \sigma_{R}=0.0625,(\bigcirc)$. The fluid field is discretized with $N=192^{3}$ Fourier modes (open symbols) in a periodic box $L_{B}=2 \pi$. In the case $d_{p} / \sigma_{R}=0.0625$, ( $)$ the fluid field is discretized with $N=24^{3}$ Fourier modes to check grid-independence. Right panel: particle velocity provided by the PIC approach in comparable conditions as in the ERPP. $d_{p} / D x=0.5$, $(\square) ; d_{p} / D x=0.25,(\triangle) ; d_{p} / D x=0.125,(\diamond) ; d_{p} / D x=0.0625,(\bigcirc)$. In both panels the reference solution (44) is reported for comparison (solid line).

grids, namely $N=192^{3}$ and $N=24^{3}$ Fourier modes, give practically undistinguishable results. It's worth noting that the error in the particle velocity is already below $10 \%$ for the relatively large ratio $d_{p} / \sigma_{R}=0.5$ we have considered. The right panel of figure 11 reports the particle velocity calculated with the PIC method. The solution presents now larger deviations from the exact result (44). This is due to a poor estimate of the hydrodynamic force. In fact, in the PIC approach the self-induced disturbance produced by the $p$ th particle is unknown or, if eventually modeled by the steady Stokeslet, is singular at the particle position $\mathbf{x}_{p}$. In both cases it can not be removed from the particle-to-fluid slip velocity resulting in an inaccurate prediction of the hydrodynamic force and, consequently, of the particle trajectory. For instance, for $d_{p} / D x=0.5$ the error on the terminal velocity is $50 \%$ for the PIC approach compared to a much lower $10 \%$ for the ERPP. Clearly the error reduces as the ratio $d_{p} / D x \rightarrow 0$.

A more direct comparison between the two approaches is provided in the left panel of figure 12 where we plot the particle velocity for the largest ratio $d_{p} / \sigma_{R}=0.5$ and the smallest one $d_{p} / \sigma_{R}=0.0625$ both for the ERPP 

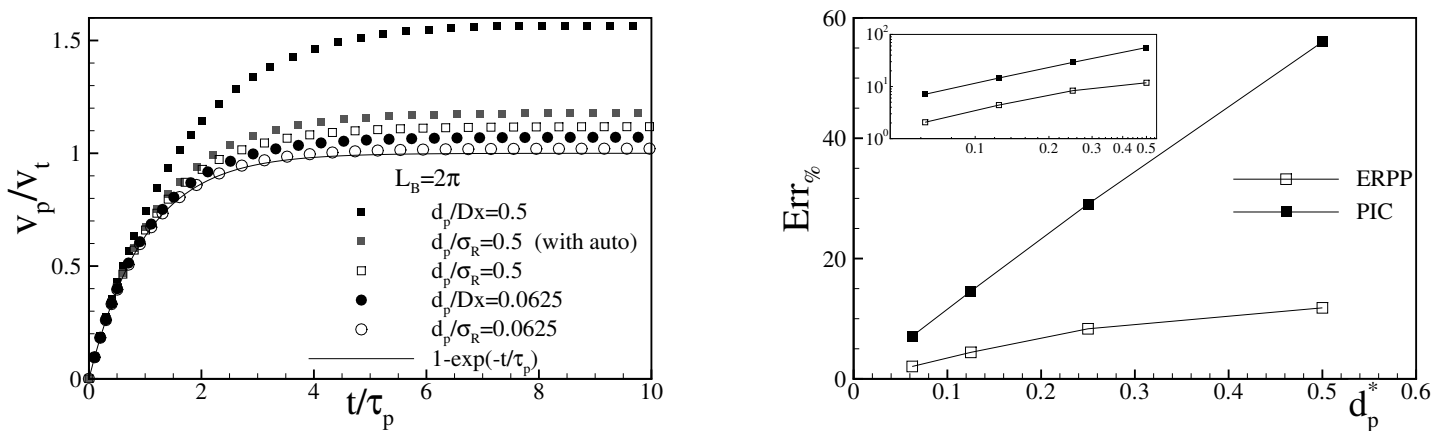

Figure 12: Normalized particle velocity $v_{p} / v_{t}$ as a function of the dimensionless time $t / \tau_{p}$. Left panel: direct comparison of the ERPP results against the PIC approach and the reference solution (solid line) for different values of the ratio $d_{p} / \sigma_{R}$ or equivalently $d_{p} / D x . d_{p} / \sigma_{R} \div d_{p} / D x=0.5$ (squares), $d_{p} / \sigma_{R} \div d_{p} / D x=0.0625$ (circles). Black filled symbols refer to the PIC approach, open symbols to the ERPP method. The grey square refers to an ERPP calculation where intentionally we did not remove the self-induced velocity disturbance. Right panel: relative error committed on the evaluation of the terminal velocity as a function of the dimensionless parameter $d_{p}^{*}=d_{p} / \sigma_{R}$ for the ERPP and the PIC. In the inset data plotted in log-log scale.

and the PIC. For comparison in the ERPP calculation we have reported the particle velocity in a case where we did not subtract from $\mathbf{u}\left(\mathbf{x}_{p}, t\right)$ the self-induced disturbance. The right panel of the figure presents the relative error committed in the estimate of the terminal velocity as a function of $d_{p} / \sigma_{R}$. Although the error scaling with grid resolution is comparable (see inset), the error pertaining to the ERPP approach is substantially smaller. A last issue concerns the sensitivity of the ERPP method in poorly resolved cases where $\sigma_{R}<D x$. In figure 13 we compare the particle velocity for three different resolutions at fixed $d_{p} / \sigma_{R}$. As expected, the method loses accuracy as the regularization kernel is not resolved on the computational grid.

\section{Application to turbulent flows}

In order to discuss the feasibility of turbulent, particle-laden flow simulations, in this section we present preliminary results obtained by the ERPP 


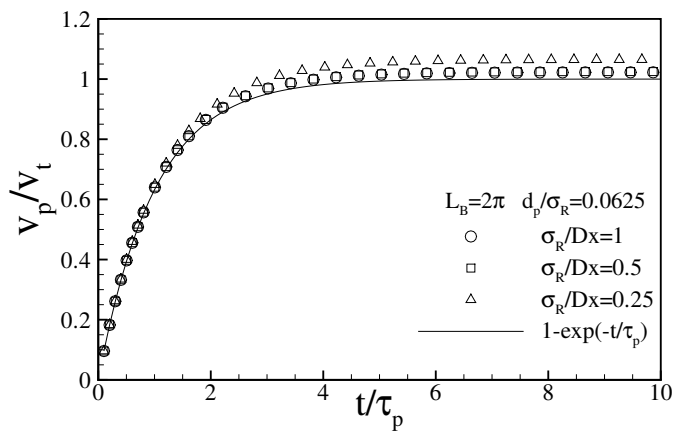

Figure 13: Normalized particle velocity $v_{p} / v_{t}$ as a function of the dimensionless time $t / \tau_{p}$. The trajectories obtained in two unresolved cases, namely $\sigma_{R} / D x=0.5$ and $\sigma_{R} / D x=0.25$ are compared against a resolved case at $\sigma_{R} / D x=1$ for a given value of the ratio $d_{p} / \sigma_{R}=0.0625$.

method for a homogeneous shear flow at moderate Reynolds number. The mean velocity profile in the $x$-direction (streamwise direction) is imposed and is characterized by a constant velocity gradient $S$ along the $y$-direction (shear direction). The third coordinate is denoted with $z$ (spanwise direction). The Reynolds decomposition $\mathbf{u}=S y \mathbf{e}_{x}+\mathbf{u}^{\prime}$ allows to write the Navier-Stokes equations for the turbulent fluctuating component $\mathbf{u}^{\prime}$ which are solved in a reference frame advected by the mean flow, see e.g. [38]. The Rogallo's transformation allows to restore the spatial homogeneity of the fluctuations in the convected frame. A sketch of the flow domain is reported in figure 14 .

In the homogeneous shear flow the turbulent fluctuations are sustained by the off-diagonal component of the Reynolds shear stress $-\langle u v\rangle$ resulting in a neat turbulent kinetic energy production rate $\mathcal{P}=-S\langle u v\rangle$. The large scale anisotropic forcing feeds the energy cascade operated by the non-linear terms of the Navier-Stokes equations which eventually restore isotropy at smaller scales. The so called shear scale $L_{S}$ ideally separates the production range $L_{S}<\ell<L_{0}$ ( $L_{0}$ is the integral scale), from the isotropy recovery range $\eta<\ell<L_{S}$. It follows that the nature of turbulent fluctuations is parametrized by two dimensionless parameter, the shear intensity $S^{*}=$ $\left(L_{0} / L_{S}\right)^{2 / 3}$ and the Corsin parameter $S_{c}=\left(\eta / L_{S}\right)^{2 / 3}$. The latter can be recast in terms of the inverse of the classical turbulent Reynolds number $R e_{\lambda}$ based on the Taylor length-scale.

In the conditions discussed above the transport of inertial particles is non 


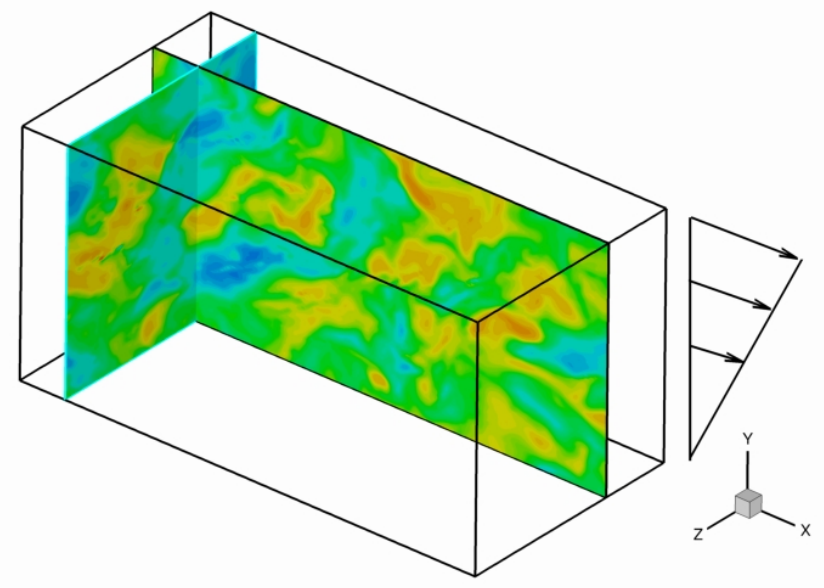

Figure 14: Sketch of the flow configuration. The flow domain is represented by a periodic box of length $L_{x}=4 \pi, L_{y}=2 \pi$ and $L_{z}=2 \pi$ in the steamwise, shear and span wise directions respectively. The mean flow $S y \mathbf{e}_{x}$ is in the $x$ direction and linearly changes at a rate $S$ along the $y$-direction. The contour plot shows the intensity of the velocity fluctuations in selected coordinate planes.

trivial. In fact, the disperse phase is characterized by small scales aggregates (clusters) which preserve a spatial preferential orientation induced by the large scale motions, up to the smallest scales where, in contrast, turbulent fluctuations recover isotropy, see e.g. [16, 17.

The first result concerns a flow at a Taylor based Reynolds number of $R e_{\lambda}=60$ and shear intensity $S^{*}=7$. The carrier phase is resolved by using $N_{x} \times N_{y} \times N_{z}=256 \times 256 \times 128$ Fourier modes in a $4 \pi \times 2 \pi \times 2 \pi$ periodic box. Such spectral-based discretization corresponds to $384 \times 384 \times 192$ collocation points in physical space due to the $3 / 2$ dealiasing procedure required for the calculation of the non linear terms. The spatial discretization fully resolves the Kolmogorov length scale with $D x / \eta \sim 1.07$. Time integration is performed by the low-storage Runge-Kutta method already mentioned in section 93 . The carrier fluid is laden with $N_{p}=2200000$ inertial particles. The particle to fluid density ratio is $\rho_{p} / \rho_{f}=1800$ corresponding to a Stokes number $S t_{\eta}=\tau_{p} / \tau_{\eta}=1$ where $\tau_{p}=\left(\rho_{p} / \rho_{f}\right) d_{p}^{2} / 18 \nu$ is the Stokes relaxation time and $\tau_{\eta}$ is the Kolmogorov time scale. In such conditions the particle diameter $d_{p}$ is much smaller than the Kolmogorov length, namely $d_{p} / \eta=0.1$. 
The mass load $\Phi$ defined as the ratio between the mass of the disperse phase and the carrier fluid is $\Phi=0.4$.

In figure 15 we present a snapshot of the particle position in a $x y$ plane containing the mean flow (from left to right). As expected, particles with unitary Stokes number are characterized by small scale clusters, i.e. the particles concentrate in narrow regions, the clusters, separated by voids where neither a particle can be found. The preferential alignment of the aggregates along the principal strain direction of the mean flow is also evident from the snapshot. This is the signature of the persistent anisotropy of the clusters at small scales. In the context of the ERPP methodology we are able to compute in a closed form the forcing operated by the particles on the fluid. In the middle panel of figure 15 we report the intensity of the forcing term of equations (34) which accounts for the back-reaction on the fluid. The pattern of the back-reaction field is strongly correlated to the cluster structure and inherit from the latter its characteristic multi-scale nature. The forcing is actually active in a broad range of scales up to the smallest scales where intense peaks occur. Note however, that the forcing field is everywhere smooth and can be successfully represented on the discrete grid by virtue of the regularization naturally operated by the viscosity. The highest forcing intensity is localized in the spatial regions where the particles concentrate while in the void regions the forcing vanishes. The correlation between the instantaneous particles spatial configuration and the corresponding backreaction on the fluid can be visually appreciated in the bottom panel of the figure where the two fields are superimposed.

As anticipated, this short paragraph is aimed at the clear illustration of the potential of the ERPP in dealing with actual turbulent flows laden with millions of particles. Clearly a complete analysis of the turbulence modulation in the two-way coupling regime would require a more complete statistical analysis which is however beyond the intents of the present work and is postponed to future investigation.

\section{$5 \quad$ Final remarks}

In this paper we have presented a new methodology, dubbed the ERPP method, able to capture the inter-phase momentum exchange between a carrier flow and a disperse phase modeled as lumped massive points. The coupling mechanism is designed on the physical ground provided by the unsteady Stokes flow around a small sphere. In short, along its trajectory the particle continuously generates a highly localized vorticity field that can 


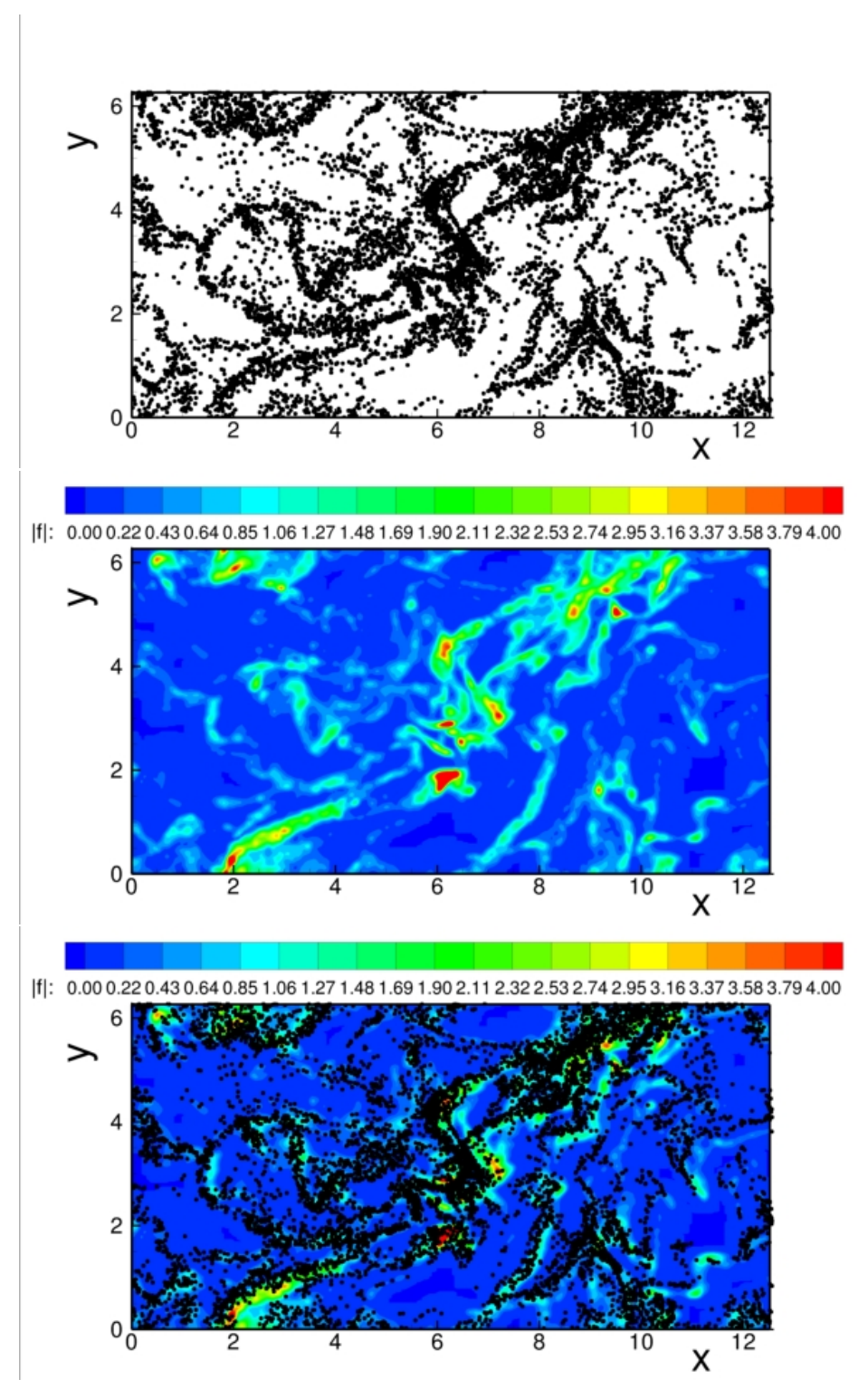

Figure 15: Snapshot of the instantaneous particle configuration (top) and corresponding intensity of the forcing on the fluid (center) in a thin slice along the $x y$ plane. The mean flow $S y$ is in the $x$ direction from left to right. The two top panels are superimposed in the bottom panel to provide a visual correlation between the instantaneous particles configuration and the corresponding forcing field. 
be evaluated in a closed form. Successively, because of viscous diffusion, the vorticity field reaches the physically significant length-scales of the flow field. When this occurs, the newly generated vorticity can be injected on the computational grid where the Navier-Stokes equations for the carrier flow are solved thus achieving the inter-phase momentum coupling. Under this respect the viscous diffusion naturally regularizes the disturbance flow produced by each particle without requiring any "ad hoc" numerical artifact. The proposed approach can be implemented in a highly efficient computational algorithm since the disturbance produced by a particle is strongly compact in space and localized around the actual particle position. This means that at each time step only few grid points perceive the particle disturbance which decays exponentially fast in space. As a consequence the ERPP method can handle millions of particles at an affordable computational cost as proved by preliminary results of a particle laden turbulent homogeneous shear flow in the two-way coupling regime.

The ERPP overcomes several drawbacks of established methods, like the Particle In Cell method. Indeed, the regularization of the back-reaction field provided by the viscous diffusion allows numerically convergent solutions, preventing the strong grid-dependence which spoils singularity-based approximations. Even more important, the ERPP method solves the intrinsic difficulty of numerical simulations in the two-way coupling regime associated with the calculation of the correct particle-to-fluid slip velocity. Actually, in the ERPP method the disturbance flow produced by the particles at each time step can be evaluated in a closed form. This allows to remove from the particle-to-fluid slip velocity the spurious self-induced velocity disturbance allowing for a correct evaluation of the hydrodynamic force.

The preliminary results concerning a turbulent particle-laden shear flow presented in the last section demonstrate the potential of the ERPP method in the simulation of turbulent flows in the two-way coupling regime. It is known that the dynamics of the two phase system is fully characterized by a given set of dimensionless parameters, namely $\left\{R e_{0}, S t_{\eta}, \rho_{p} / \rho_{f}, d_{p} / \eta, \Phi, N_{p}\right\}$. To comment on the effectiveness of the ERPP method in modeling turbulent suspensions, let us assume that the turbulence characteristics are prescribed i.e. the the turbulent Reynolds number $R e_{0}$, the integral scale $L_{0}$, the Kolmogorov scale $\eta$ or timescale $\tau_{\eta}$ are fixed, and let us consider small particles, i.e. $d_{p} / \eta \ll 1$. In these conditions the Stokes number $S t_{\eta}=\tau_{p} / \tau_{\eta}$ controls the dynamics of the disperse phase in terms of its preferential spatial accumulation, either small scale clustering in homogeneous flows [42, 28, 4, 29, 37. or turbophoresis in wall bounded flows [34, 25]. Once the Stokes number is 
fixed, the mass load $\Phi$ follows as

$$
\Phi=\frac{\pi}{6} N_{p} \frac{\left(18 S t_{\eta}\right)^{3 / 2}}{\left(\rho_{p} / \rho_{f}\right)^{1 / 2} R e_{0}^{9 / 4}} .
$$

The value of $\Phi$ can be adjusted by means of the density ratio $\rho_{p} / \rho_{f}$ and the number of particles $N_{p}$. However, in an actual experiment the ratio $\rho_{p} / \rho_{f}$ must fall in the range of the available materials and the most straightforward way to achieve the desired mass load consists in adjusting the number of particles $N_{p}$. Although rather easy in experiments, adjusting the number of particles turns out to be a big issue in numerical simulations since, most often, the momentum coupling model is unable to handle an arbitrary number of particles while providing grid-independent and physically consistent results. At variance with most available methods, both these requirements are fulfilled by the ERPP approach. Indeed, the number of particles can be freely changed since disturbance flow and back-reaction of each particle are smooth fields. This implies that the solution is correctly reproduced also in flow regions where the particles are extremely dilute, like it happens for the exterior region of turbulent jets or spatially evolving boundary layers. For comparison, classical approaches like the PIC method intrinsically suffer of spurious numerical oscillations in the back-reaction field when too few particles per computational cell are available, leading to strong limitations in the achievable mass load $\Phi$. Indeed, in any Direct Numerical Simulation of a turbulent flow the number of computational cells scales with the Reynolds number like $N_{c} \sim R e_{0}^{9 / 4}$ suggesting through equation (47) that no room is available to adjust the mass load if the additional constraint $N_{p} / N_{c} \sim 1$ needs to be enforced. This limitation is overcome in the approach proposed here by relaxing the request on the particle density to allow for the modeling freedom needed to reproduce any physically relevant condition.

\section{Ackonwledgements}

The results of this research have been achieved using the PRACE-2IP project (FP7 RI-283493) resource Zeus based in Poland at Krakow. The authors are gratefull to the COST Action MP0806 Particles in Turbulence. 


\section{A}

\section{A.1 Fundamental solution of the diffusion equation}

The fundamental solution of the diffusion equation $g(\mathbf{x}-\boldsymbol{\xi}, t-\tau)$ can be found by solving the following singularily forced diffusion problem

$$
\frac{\partial g}{\partial t}-\nu \nabla^{2} g=\delta(\mathbf{x}-\boldsymbol{\xi}) \delta(t-\tau)
$$

with $\lim _{t \rightarrow \tau^{-}} g(\mathbf{x}-\boldsymbol{\xi}, t-\tau)=g\left(\mathbf{x}-\boldsymbol{\xi}, 0^{-}\right)=0$ expressing the causality principle. By integrating equation (48) in the interval $[\tau-\epsilon, \tau+\epsilon]$ and letting $\epsilon$ approach zero, the singularly forced diffusion equation is recast into an initial value problem

$$
\begin{aligned}
& \frac{\partial g}{\partial t}-\nu \nabla^{2} g=0 \quad t>\tau \\
& \lim _{t \rightarrow \tau^{+}} g(\mathbf{x}-\boldsymbol{\xi}, t-\tau)=g\left(\mathbf{x}-\boldsymbol{\xi}, 0^{+}\right)=\delta(\mathbf{x}-\boldsymbol{\xi}),
\end{aligned}
$$

whose solution is immediate in Fourier space. By denoting with $\hat{g}(\mathbf{k}, t-\tau)$ the Fourier transform of $g(\mathbf{x}-\boldsymbol{\xi}, t-\tau)$, equation 49$)$ reads

$$
\begin{aligned}
& \frac{\partial \hat{g}}{\partial t}+\nu\|\mathbf{k}\|^{2} \hat{g}=0 \quad t>\tau \\
& \lim _{t \rightarrow \tau^{+}} \hat{g}(\mathbf{k}, t-\tau)=\hat{g}\left(\mathbf{k}, 0^{+}\right)=\frac{1}{(2 \pi)^{3}} .
\end{aligned}
$$

The solution in Fourier space is

$$
\hat{g}(\mathbf{k}, t-\tau)=\frac{1}{(2 \pi)^{3}} \exp \left[-\nu\|\mathbf{k}\|^{2}(t-\tau)\right],
$$

which, after inverse Fourier transformation, yields the fundamental solution

$$
g(\mathbf{x}-\boldsymbol{\xi}, t-\tau)=\frac{1}{[4 \pi \nu(t-\tau)]^{3 / 2}} \exp \left[-\frac{\|\mathbf{x}-\boldsymbol{\xi}\|^{2}}{4 \nu(t-\tau)}\right]
$$

as a Gaussian function with time dependent variance $\sigma(t-\tau)=\sqrt{2 \nu(t-\tau)}$. 


\section{A.2 Fundamental solution of the unsteady Stokes equations}

The fundamental solution of the unsteady Stokes operator can be found by solving the singularly forced unsteady Stokes equations, namely

$$
\begin{aligned}
& \nabla \cdot \mathbf{v}=0 \\
& \rho_{f} \frac{\partial \mathbf{v}}{\partial t}=-\nabla p+\mu \nabla^{2} \mathbf{v}+\hat{\mathbf{e}} \delta(\mathbf{x}-\boldsymbol{\xi}) \delta(t-\tau) \\
& \lim _{t \rightarrow \tau^{-}} \mathbf{v}(\mathbf{x}-\boldsymbol{\xi}, t-\tau)=\mathbf{v}\left(\mathbf{x}-\boldsymbol{\xi}, 0^{-}\right)=0
\end{aligned}
$$

where the sigular forcing $\delta(\mathbf{x}-\boldsymbol{\xi}) \delta(t-\tau)$ is applied at the point $\mathbf{x}=\boldsymbol{\xi}$ at time $t=\tau$ along the direction $\hat{\mathbf{e}}$. The solution of equations (53) is more easily found in terms of the associtaed vorticity field $\zeta=\nabla \times \mathbf{v}$. By taking the curl of equations $(53)$ it follows

$$
\rho_{f} \frac{\partial \boldsymbol{\zeta}}{\partial t}=\mu \nabla^{2} \boldsymbol{\zeta}-\hat{\mathbf{e}} \times \nabla \delta(\mathbf{x}-\boldsymbol{\xi}) \delta(t-\tau)
$$

with the corresponding initial condition $\boldsymbol{\zeta}\left(\mathbf{x}-\boldsymbol{\xi}, 0^{-}\right)=0$. Equation (54) can be reconducted to the standard scalar diffusion equation (48) by the ansatz

$$
\zeta=-\frac{1}{\rho_{f}} \hat{\mathbf{e}} \times \nabla g .
$$

Equations (55) and 52 provides the solution of the singularly forced unsteady Stokes problem in terms of vorticity. The solution in terms of velocity can be found by introducing a divergence free vector potential A, namely $\mathbf{v}=\nabla \times \mathbf{A}$ and $\nabla^{2} \mathbf{A}=-\boldsymbol{\zeta}$. The Laplace equation for the vector potential can be transformed into a scalar equation by looking for solutions for the vector potential in the form

$$
\mathbf{A}=\frac{1}{\rho_{f}} \hat{\mathbf{e}} \times \nabla G,
$$

where the scalar function $G$ satisfy the standard Laplace equation $\nabla^{2} G=g$. The solution for $G$ reads

$$
G=-\frac{1}{4 \pi r} \operatorname{erf}\left(\frac{r}{\sqrt{4 \nu(t-\tau)}}\right)
$$

where $r=\|\mathbf{x}-\boldsymbol{\xi}\|$. The velocity field can be readily detemined by substituting the expressions (56) and (57) into $\mathbf{v}=\nabla \times \mathbf{A}$. After some algebra the velocity reads

$$
\mathbf{v}=(g \mathbf{I}-\nabla \otimes \nabla G) \hat{\mathbf{e}} .
$$


The solution (58) is usually written in terms of the Green tensor $G_{i k}(\mathbf{x}-$ $\boldsymbol{\xi}, t-\tau)$. In fact by using (52) and (57), equation (58) can be written as

$$
v_{i}=G_{i k} \hat{e}_{k}
$$

where the Green tensor is given by the expresion

$$
G_{i k}(\mathbf{x}-\boldsymbol{\xi}, t-\tau)=\frac{1}{\rho_{f}}\left[\left(1+\frac{\sigma^{2}}{r^{2}}\right) g+\frac{G}{r^{2}}\right] \delta_{i k}-\frac{1}{\rho_{f}}\left[\left(1+\frac{3 \sigma^{2}}{r^{2}}\right) g+\frac{3 G}{r^{2}}\right] \frac{r_{i} r_{k}}{r^{2}} .
$$

The solution of the singularly forced Stokes problem is completed by the expression of the pressure and of the viscous stressed. The pressure field associeted to the original problem (53) can be computed by taking the divergence of the momentum equation, namely

$$
\nabla^{2} p=\hat{\mathbf{e}} \cdot \nabla \delta(\mathbf{x}-\boldsymbol{\xi}) \delta(t-\tau) .
$$

The Laplace equation for the pressure can be readily solved by the substitution $p=\hat{\mathbf{e}} \cdot \nabla q \delta(t-\tau)$. In fact, the function $q$ satisfies the Laplace problem $\nabla^{2} q=\delta(\mathbf{x}-\boldsymbol{\xi})$, i.e. $q=-\frac{1}{4 \pi r}$. The pressure field then follows at once as

$$
p=\frac{\hat{\mathbf{e}} \cdot \mathbf{r}}{4 \pi r^{3}} \delta(t-\tau) \text {. }
$$

The stress tensor associated to the singularly forced unsteady Stokes problem can be computed as

$$
T_{i j}=-p \delta_{i j}+\mu\left(\frac{\partial v_{i}}{\partial x_{j}}+\frac{\partial v_{j}}{\partial x_{i}}\right)
$$

where the pressure $p$ is given by $(62)$ and the velocity $\mathbf{v}$ by $(59)$. Usually the stress tensor is expressed via a third rank tensor

$$
T_{i j}=\mathcal{T}_{i j k} \hat{e}_{k}
$$

where $\mathcal{T}_{i j k}$ is the Green stress tensor defined as

$$
\mathcal{T}_{i j k}=-\frac{r_{k}}{4 \pi r^{3}} \delta(t-\tau) \delta_{i j}+\mu\left(\frac{\partial G_{i k}}{\partial x_{j}}+\frac{\partial G_{j k}}{\partial x_{i}}\right) .
$$

The expression of the Green tensor (60) can be substituted into the definition (65) and, after some algebra, the final expression for $\mathcal{T}_{i j k}$ reads

$$
\begin{aligned}
\mathcal{T}_{i j k} & =-\frac{r_{k}}{4 \pi r^{3}} \delta(t-\tau) \delta_{i j} \\
& +\nu\left[-2 \frac{B}{r^{2}} \delta_{i j} r_{k}+\left(\frac{1}{r} \frac{d A}{d r}-\frac{B}{r^{2}}\right)\left(\delta_{j k} r_{i}+\delta_{i k} r_{j}\right)-2\left(\frac{2}{r} B+\frac{d B}{d r}\right) \frac{r_{i} r_{j} r_{k}}{r^{3}}[6]\right.
\end{aligned}
$$


where the functions $A(r)$ and $B(r)$ are defined as

$$
\begin{aligned}
& A(r)=\left(1+\frac{\sigma^{2}}{r^{2}}\right) g+\frac{G}{r^{2}} \\
& B(r)=\left(1+\frac{3 \sigma^{2}}{r^{2}}\right) g+\frac{3}{r^{2}} G
\end{aligned}
$$

\section{A.3 Evolution of the regular vorticity field, proof of equation} (21)

Let's first differentiate equation (17) with respect to time,

$$
\begin{aligned}
\frac{\partial \boldsymbol{\zeta}_{R}}{\partial t} & =\frac{1}{\rho_{f}} \mathbf{D}_{p}\left(t-\epsilon_{R}\right) \times \nabla_{x} g\left[\mathbf{x}-\mathbf{x}_{p}\left(t-\epsilon_{R}\right), \epsilon_{R}\right] \\
& +\frac{1}{\rho_{f}} \int_{0}^{t-\epsilon_{R}} \mathbf{D}_{p}(\tau) \times \nabla_{x} \frac{\partial g}{\partial t}\left[\mathbf{x}-\mathbf{x}_{p}(\tau), t-\tau\right] d \tau
\end{aligned}
$$

take the Laplacian,

$$
\nabla^{2} \boldsymbol{\zeta}_{R}=\frac{1}{\rho_{f}} \int_{0}^{t-\epsilon_{R}} \mathbf{D}_{p}(\tau) \times \nabla_{x} \nabla^{2} g\left[\mathbf{x}-\mathbf{x}_{p}(\tau), t-\tau\right] d \tau,
$$

and recombine the two results with the kinematic viscosity yielding

$$
\begin{aligned}
\frac{\partial \boldsymbol{\zeta}_{R}}{\partial t}-\nu \nabla^{2} \boldsymbol{\zeta}_{R} & =\frac{1}{\rho_{f}} \mathbf{D}_{p}\left(t-\epsilon_{R}\right) \times \nabla_{x} g\left[\mathbf{x}-\mathbf{x}_{p}\left(t-\epsilon_{R}\right), \epsilon_{R}\right] \\
& +\frac{1}{\rho_{f}} \int_{0}^{t-\epsilon_{R}} \mathbf{D}_{p}(\tau) \times \nabla_{x}\left\{\frac{\partial g}{\partial t}-\nu \nabla^{2} g\right\}\left[\mathbf{x}-\mathbf{x}_{p}(\tau), t-\tau\right] d \tau \\
& =\frac{1}{\rho_{f}} \mathbf{D}_{p}\left(t-\epsilon_{R}\right) \times \nabla_{x} g\left[\mathbf{x}-\mathbf{x}_{p}\left(t-\epsilon_{R}\right), \epsilon_{R}\right] \\
& +\frac{1}{\rho_{f}} \int_{0}^{t-\epsilon_{R}} \mathbf{D}_{p}(\tau) \times \nabla_{x} \delta\left[\mathbf{x}-\mathbf{x}_{p}(\tau)\right] \delta(t-\tau) d \tau \\
& =\frac{1}{\rho_{f}} \mathbf{D}_{p}\left(t-\epsilon_{R}\right) \times \nabla_{x} g\left[\mathbf{x}-\mathbf{x}_{p}\left(t-\epsilon_{R}\right), \epsilon_{R}\right]
\end{aligned}
$$

\section{A.4 Singular part of the velocity field}

The contribution of the singular component of the velocity disturbance $\mathbf{v}_{S}$ due to the particles can be estimated starting from the expression of the 
associated singular vorticity field given in equation (18) that we report here for convenience, namely

$$
\boldsymbol{\zeta}_{S}(\mathbf{x}, t)=\frac{1}{\rho_{f}} \int_{t-\epsilon_{R}}^{t^{+}} \mathbf{D}_{p}(\tau) \times \nabla g\left[\mathbf{x}-\mathbf{x}_{p}(\tau), t-\tau\right] d \tau
$$

The time integral for small values of $\epsilon_{R}$ can be approximated as

$$
\boldsymbol{\zeta}_{S}(\mathbf{x}, t)=\frac{\mathbf{D}_{p}^{*}}{\rho_{f}} \times \nabla \int_{t-\epsilon_{R}}^{t^{+}} g\left[\mathbf{x}-\mathbf{x}_{p}\left(\tau^{*}\right), t-\tau\right] d \tau .
$$

where $\mathbf{D}_{p}^{*}=\sup _{t-\epsilon_{R}<\tau<t^{+}} \mathbf{D}_{p}(\tau)$ and $\tau^{*}$ is the time corresponding to the minimum distance between the actual particle position $\mathbf{x}_{p}\left(\tau^{*}\right)$ and the point $\mathbf{x}$. The time integral in equation (71) can be explicitly computed leading to the following expression of the singular vorticity field

$$
\boldsymbol{\zeta}_{S}=\mathbf{D}_{p}^{*} \times \nabla H \quad \text { with } \quad H=\frac{1}{4 \pi \mu r}\left[1-\operatorname{erf}\left(\frac{r}{\sqrt{2} \sigma_{R}}\right)\right],
$$

where $r=\left|\mathbf{x}-\mathbf{x}_{p}\left(\tau^{*}\right)\right|$. Given the vorticity, the corresponding velocity can be found in terms of the associated divergence free vector potential, namely $\mathbf{v}_{S}=\nabla \times \mathbf{A}_{S}$, by solving the Poisson problem $\nabla^{2} \mathbf{A}_{S}=-\boldsymbol{\zeta}_{S}$. The solution can be found in the form $\mathbf{A}_{S}=-\mathbf{D}_{p}^{*} \times \nabla \psi$ where $\psi$ is the solution of the scalar problem $\nabla^{2} \psi=H$. The singular velocity field is then expressed as

$$
\mathbf{v}_{S}(\mathbf{x}, t)=\left(\nabla \otimes \nabla \psi-\nabla^{2} \psi\right) \mathbf{D}_{p}^{*}
$$

and $\psi$ is given by

$$
\psi=\frac{\sqrt{2} \sigma_{R}}{8 \pi \mu}\left[\eta-\eta \operatorname{erf}(\eta)-\frac{1}{2 \eta} \operatorname{erf}(\eta)-\frac{1}{\sqrt{\pi}} \exp \left(-\eta^{2}\right)\right],
$$

in terms of the dimensionless variable $\eta=r / \sqrt{2} \sigma_{R}$. The explicit expression of $\mathbf{v}_{S}$ is only a matter of successive derivation of expression (74). After calculations, the singular velocity field can be finally expressed as

$$
\mathbf{v}_{i}^{S}(\mathbf{x}, t)=\frac{D_{j}^{p *}}{8 \pi \mu}\left[\left(\frac{\partial^{2} \psi}{\partial \eta^{2}}-\frac{1}{\eta} \frac{\partial \psi}{\partial \eta}\right) \frac{r_{i} r_{j}}{r^{2}}-\left(\frac{\partial^{2} \psi}{\partial \eta^{2}}+\frac{1}{\eta} \frac{\partial \psi}{\partial \eta}\right) \delta_{i j}\right] .
$$

Expression (75) is amenable of further manipulation to extract the near field behavior of the singular velocity field, i.e. the expression of $\mathbf{v}_{S}$ in the limit of $\eta \rightarrow 0$. In fact, for small values of $\eta$, the error function which appears 
in $\psi$ and in its first and second derivatives can be expanded in McLaurin series. After some algebra, equation (75) can be recasted in the form

$$
\mathbf{v}_{i}^{S}(\mathbf{x}, t)=-\frac{D_{j}^{p *}}{8 \pi \mu r}\left(\delta_{i j}+\frac{r_{i} r_{j}}{r^{2}}\right)
$$

which express the behavior of the singular field for small distances $r$ from the particle when compared with the diffusion length-scale $\sigma_{R}$. From equation (76) it appears that the singular velocity field still presents a singularity which diverges as $1 / r$ in the neighborhood of the actual particle position $\mathbf{x}_{p}$. In principle, the singular velocity field gives a finite contribution to the convective terms of the Navier-Stokes equations, see e.g. equations (34). By coarse graining the equations on a scale $\Delta$, small with respect to the hydrodynamic scale but larger than the particle size one can show that the contribution arising from $\mathbf{v}_{S} \cdot \nabla \mathbf{v}_{S}, \mathbf{v}_{S} \cdot \nabla \mathbf{u}_{R}$ and $\mathbf{u}_{R} \cdot \nabla \mathbf{v}_{S}$ are negligibly small and can be neglected. In performing the coarse graining, one has to consider that the convolution integral should be performed in the region occupied by the fluid, i.e. outside the particles. For instance, let us refer to the sketch in figure 16 where the particle placed at $\mathbf{x}_{p}$ induces a velocity disturbance in $\mathbf{y}$ and the coarse grained velocity field is evaluated at point $\mathbf{x}$. In the relative position of the particle with respect to the point $\mathbf{x}$ we will discuss two typical cases. The particle can lay entirely inside the region where the filter kernel is non vanishing or it can partially lay outside the filter kernel radius. Lastly the particle might lay completely outside the filter kernel. In all cases the coarse grained advective terms at point $\mathbf{x}$ can be computed as a convolution of the relevant part of the convective term with a filter Kernel $K$, e.g.

$$
\mathbf{h}_{S S}(\mathbf{x}, t)=\int_{\Omega_{\Delta} \backslash \Omega_{p}} \mathbf{v}_{S}(\mathbf{y}, t) \cdot \nabla_{\mathbf{y}} \mathbf{v}_{S}(\mathbf{y}, t) K(\mathbf{y}-\mathbf{x}) d^{3} \mathbf{y}
$$

where the integration variable $\mathbf{y}$ belongs to the domain $\Omega_{\Delta} \backslash \Omega_{p}$ which is the complement to the support $\Omega_{\Delta}$ of the filter of the region $\Omega_{p}$ occupied by the particle. By assuming, for the sake of definiteness, a top-hat kernel,

$$
K(\mathbf{y}-\mathbf{x})=\left\{\begin{array}{cc}
\frac{1}{\Delta^{3}} & |\mathbf{y}-\mathbf{x}|<\Delta \\
0 & |\mathbf{y}-\mathbf{x}|>\Delta
\end{array}\right.
$$

the convolution integral (77) can be transformed by incompressibility of the field $\mathbf{v}_{S}$ into a surface integral

$$
\mathbf{h}_{S S}(\mathbf{x}, t)=\frac{1}{\Delta^{3}} \int_{\partial\left(\Omega_{\Delta} \backslash \Omega_{p}\right)} \mathbf{v}_{S}\left(\mathbf{v}_{S} \cdot \mathbf{n}\right) d S_{\mathbf{y}}
$$



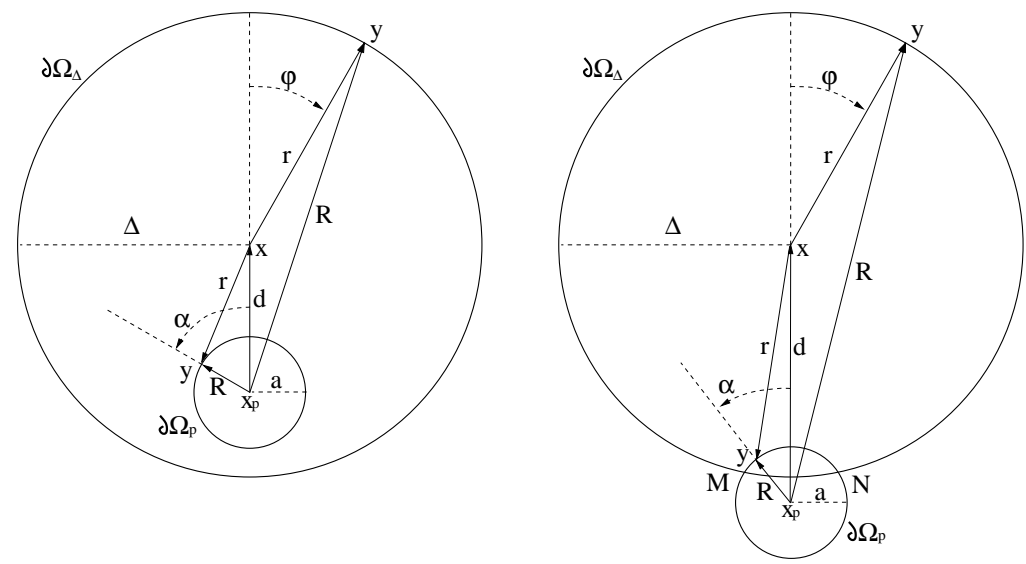

Figure 16: Sketch of the coarse-graining procedure in the neigbourhood of the Eulerian point $\mathbf{x}$. The boundary $\partial \Omega_{\Delta}$ denotes the fileter kernel of width $\Delta$ and $\partial \Omega_{p}$ is the particle spherical surface of radius $a<\Delta$. Two cases are possible. The particle entirely lays inside the region where the filter kernel is non vanisshing (left), or the particle lays partially ouside the filter radius $\Delta$.

In expression $(79)$ the integration point $\mathbf{y}$ runs on $\partial \Omega_{\Delta} \cup \partial \Omega_{p}$ when the particle lays entirely within the filter width, right panel of figure 16 . In the other case when the particle partially intercepts the filter boundary, see the right sketch of the figure, the point $\mathbf{y}$ runs on $\left(\partial \Omega_{p} \cap \Omega_{\Delta}\right) \cup \partial \Omega_{\Delta} \backslash\left(\partial \Omega_{\Delta} \cap \Omega_{p}\right)$. For detailed calculations it is convenient to define the vectors $\mathbf{r}=\mathbf{y}-\mathbf{x}$, $\mathbf{R}=\mathbf{y}-\mathbf{x}_{p}$ and $\mathbf{d}=\mathbf{x}-\mathbf{x}_{p}$. In fact, when $\mathbf{y} \in \partial \Omega_{\Delta}$ the integration in 79 is better evaluated in terms of the $\mathbf{r}$ variable while for $\mathbf{y} \in \partial \Omega_{p}$ the use of the integration variable $\mathbf{R}$ ease the calculations. In particular for $\mathbf{y} \in \partial \Omega_{\Delta}$ the outward positive normal is $\mathbf{n}=\hat{\mathbf{r}}$ where the hat denote $\hat{\mathbf{r}}=\mathbf{r} / r$ while for $\mathbf{y} \in \partial \Omega_{p}$ the positive normal is $\mathbf{n}=-\hat{\mathbf{R}}$. Note that in the new variables the singular velocity field depends on $\mathbf{R}, \mathbf{v}_{S}(\mathbf{R}, t)$ with $\mathbf{R}=\mathbf{d}+\mathbf{r}$. By exploiting the expression $(76)$ for $\mathbf{v}_{S}$, the integrand function in equation $(79)$ for $\mathbf{y} \in \partial \Omega_{\Delta}$ is

$$
\begin{aligned}
\mathbf{v}_{S}\left(\mathbf{v}_{S} \cdot \mathbf{n}\right) & =\frac{1}{(8 \pi \mu R)^{2}}\left\{\left[\left(\mathbf{D}_{p}^{*} \cdot \hat{\mathbf{r}}\right)+\left(\mathbf{D}_{p}^{*} \cdot \hat{\mathbf{R}}\right)(\hat{\mathbf{R}} \cdot \hat{\mathbf{r}})\right] \mathbf{D}_{p}^{*}\right. \\
& \left.+\left(\mathbf{D}_{p}^{*} \cdot \hat{\mathbf{R}}\right)\left[\left(\mathbf{D}_{p}^{*} \cdot \hat{\mathbf{r}}\right)+\left(\mathbf{D}_{p}^{*} \cdot \hat{\mathbf{R}}\right)(\hat{\mathbf{R}} \cdot \hat{\mathbf{r}})\right] \hat{\mathbf{R}}\right\}
\end{aligned}
$$


Or

$$
\mathbf{v}_{S}\left(\mathbf{v}_{S} \cdot \mathbf{n}\right)=\frac{2}{(8 \pi \mu R)^{2}}\left(\mathbf{D}_{p}^{*} \cdot \hat{\mathbf{R}}\right)\left[\mathbf{D}_{p}^{*}+\left(\mathbf{D}_{p}^{*} \cdot \hat{\mathbf{R}}\right) \hat{\mathbf{R}}\right]
$$

when $\mathbf{y} \in \partial \Omega_{p}$.

Let us discuss the case when the particle is entirely within the filter kernel, see left panel of figure 16. The contributions to the surface integral coming from $\mathbf{y} \in \partial \Omega_{p}$ identically vanishes while the contribution from $\mathbf{y} \in$ $\partial \Omega_{\Delta}$ in equation 79 can be explicitly calculated by using a system of spherical coordinates centered in $\mathbf{x}$, i.e. by integrating with respect to $\mathbf{r}$ the expression 80 . After tedious but straightforward calculations it can be proved that each term arising from 80 gives a finite contribution to the integral thus providing the following estimate for $\mathbf{h}_{S S}$, namely

$$
\mathbf{h}_{S S} \sim \frac{\left|\mathbf{D}_{p}^{*}\right|^{2}}{\mu^{2} \Delta^{3}} f\left(\frac{d}{\Delta}\right)
$$

where $f(d / \Delta)$ is a regular function of the ratio $d / \Delta$, with $0 \leq d / \Delta<$ $1-a / \Delta$.

The same conclusion holds when the calculations are repeated for the case when the particle intercepts the filter boundary, left panel of figure 16. The mixed advective terms $\mathbf{v}_{S} \cdot \nabla \mathbf{u}_{R}$ and $\mathbf{u}_{R} \cdot \nabla \mathbf{v}_{S}$ can be calculated by means of the same procedure assuming that the regular contribution $\mathbf{u}_{R}$ and its gradients are constant on the filter length-scale $\Delta$. In such conditions, the corresponding coarse-grained contributions scale as

$$
\mathbf{h}_{R S} \sim \frac{\left|\mathbf{D}_{p}^{*}\right|^{2}}{\mu^{2} \sigma_{R} \Delta^{2}} \quad \mathbf{h}_{S R} \sim \frac{\left|\mathbf{D}_{p}^{*}\right|^{2}}{\mu^{2} \sigma_{R}^{2} \Delta} .
$$

The estimates 82 and $(83)$ can be used to compare the order of magnitude of the advective terms against the order of magnitude of the feedback term in equations (34) which scales as

$$
\frac{\mathbf{D}_{p}\left(t-\epsilon_{R}\right)}{\rho_{f}} g\left[\mathbf{x}-\mathbf{x}_{p}\left(t-\epsilon_{R}\right), \epsilon_{R}\right] \sim \frac{\left|\mathbf{D}_{p}\right|}{\rho_{f} \sigma_{R}^{3}} .
$$

From the above estimates, it follows

$$
\frac{\mathcal{O}\left(\mathbf{h}_{S S}\right)}{\mathcal{O}\left(\frac{\mathbf{D}_{p}}{\rho_{f}} g\right)} \sim R e_{p}\left(\frac{\sigma_{R}}{\Delta}\right)^{3} \quad \frac{\mathcal{O}\left(\mathbf{h}_{R S} ; \mathbf{h}_{S R}\right)}{\mathcal{O}\left(\frac{\mathbf{D}_{p}}{\rho_{f}} g\right)} \sim R e_{p}\left(\frac{\sigma_{R}}{\Delta}\right)^{2}
$$


where $R e_{p}$ is the particle Reynolds number calculated by using the particle radius $a$, the particle-to-fluid slip velocity $w_{r e l}$ and the kinematic viscosity $\nu$. It follows that all these terms can be neglected in the limit of small particle Reynolds number.

Let us give an example of the detailed calculations of the integral 79 in the two cases reported in figure 16. We first address the case reported in the left panel of the figure where the particle is entirely inside the filter kernel hence the integral is splitted on $\partial \Omega_{\Delta}$ where the integrand function is given by equation $(80)$ and $\partial \Omega_{p}$ where the expression $(81)$ must be adopted. Let us first discuss the integration of equation 80 on $\partial \Omega_{\Delta}$. For convenience we fix the polar axis along the direction $\mathbf{e}_{3}$ such that $r_{1}=r \sin \phi \cos \theta, r_{2}=$ $r \sin \phi \sin \theta, r_{3}=r \cos \phi$. Due to the symmetries of the problem only the terms involving the contributions from $r_{3}, R_{3}, R_{1}^{2} R_{3}, R_{2}^{2} R_{3}, R_{3}^{3}$ give a contribution to the integral. In the following we address the term $\left(\mathbf{D}_{p}^{*} \cdot \hat{\mathbf{r}}\right) \mathbf{D}_{p}^{*}$ wich reduces to

$$
\frac{1}{\Delta^{3}} \frac{\mathbf{D}_{p}^{*} D_{p}^{3^{*}}}{(8 \pi \mu)^{2}} \int_{\partial \Omega_{\Delta}} \frac{\hat{r_{3}}}{R^{2}} \Delta^{2} \sin \phi d \phi d \theta
$$

where $R=\sqrt{\Delta^{2}+d^{2}+2 d \Delta \cos \phi}$. By defining $b=d / \Delta$ we get

$$
\frac{2 \pi}{\Delta^{3}} \frac{\mathbf{D}_{p}^{*} D_{p}^{3^{*}}}{(8 \pi \mu)^{2}} \int_{-1}^{1} \frac{\xi}{1+b^{2}+2 b \xi} d \xi
$$

and

$$
\frac{2 \pi}{\Delta^{3}} \frac{\mathbf{D}_{p}^{*} D_{p}^{3^{*}}}{(8 \pi \mu)^{2}} \frac{1}{4 b^{2}}\left[4 b+2\left(b^{2}+1\right) \ln \frac{|b-1|}{|b+1|}\right] .
$$

The above expression holds for $0 \leq b<1-a / \Delta$ and is apparently singular for $b \rightarrow 0$. However for small values of $b$ we have $\ln |b-1|=\ln (1-b) \simeq-b$ and $\ln (1+b) \simeq b$. It follows that the term in square brackets in expression 88 goes like $-4 b^{3}$ and equation (88) vanishes for $d / \Delta \rightarrow 0$. A term which gives a finite contribution the expression 79 is indeed given by $\left(\mathbf{D}_{p}^{*} \cdot \hat{\mathbf{R}}\right)(\hat{\mathbf{R}} \cdot \hat{\mathbf{r}}) \mathbf{D}_{p}^{*}$ wich reduces to $D_{p}^{3^{*}} \hat{R}_{3}(\hat{\mathbf{R}} \cdot \hat{\mathbf{r}})$. Hence the expression is transformed into

$$
\frac{1}{\Delta^{3}} \frac{\mathbf{D}_{p}^{*} D_{p}^{3^{*}}}{(8 \pi \mu)^{2}} \int_{\partial \Omega_{\Delta}} \frac{\hat{R_{3}}(\hat{\mathbf{R}} \cdot \hat{\mathbf{r}})}{R^{2}} \Delta^{2} \sin \phi d \phi d \theta
$$

where $(\hat{\mathbf{R}} \cdot \hat{\mathbf{r}})=(1+b \cos \phi) / \sqrt{1+b^{2}+2 b \cos \phi}$. After substitution we get the integral

$$
\frac{2 \pi}{\Delta^{3}} \frac{\mathbf{D}_{p}^{*} D_{p}^{3^{*}}}{(8 \pi \mu)^{2}} \int_{-1}^{1} \frac{(1+b \xi)^{2}}{\left(1+b^{2}+2 b \xi\right)^{2}} d \xi
$$


which can be integrated providing the following expression

$$
\frac{2 \pi}{\Delta^{3}} \frac{\mathbf{D}_{p}^{*} D_{p}^{3^{*}}}{(8 \pi \mu)^{2}} \frac{1}{4 b}\left[4 b+2\left(1-b^{2}\right) \ln \frac{|b+1|}{|b-1|}\right] .
$$

For small values of $b$ we have $\ln |b+1|-\ln |b-1| \simeq 2 b$ hence the term in square bracket goes like $8 b-4 b^{2}$ resulting in a finite limit of expression (91). Let us know discuss the integration of the field given by (81) on $\partial \Omega_{p}$. The calculation is strightforward when the integral is computed with respect the variables $\mathbf{R}$. In fact, it can be prooved that each contribution arising from expression (81) vanishes in agreement with the fact that the field $\mathbf{v}_{S}$ is spherically simmetric with respect to the natural variable $\mathbf{R}$. We complete the discussion by discussing the integral (79) when the particle partially intersect the filter boundary, see the right panel of figure 16 . In this case the field 80 must be used on $\partial \Omega_{\Delta} \backslash\left(\partial \Omega_{\Delta} \cap \Omega_{p}\right)$ and the expression (81) on $\left(\partial \Omega_{p} \cap \Omega_{\Delta}\right)$. The same calculations reported above can be easily repeated by taking into account that the angle $\phi$ or $\alpha$ assume values in $\left[0: \phi_{\max }\right]$ and $\left[0: \alpha_{\max }\right]$ respectively and that the ratio $b=d / \Delta>1-a / \Delta$. Such limitations exclude any singular behaviors of the integrals both on $\partial \Omega_{\Delta} \backslash\left(\partial \Omega_{\Delta} \cap \Omega_{p}\right)$ and $\left(\partial \Omega_{p} \cap \Omega_{\Delta}\right)$.

\section{A.5 Evaluation of the self-induced disturbance flow}

The self-disturbance flow produced by the $p$ th particle in a generic time step $t_{n} \rightarrow t_{n+1}$ can be evaluated by integrating the complete equation for the disturbance field, namely equation (25), that we report below in a slighly different notation where the subscript $R$ is omitted

$$
\frac{\partial \mathbf{v}}{\partial t}-\nu \nabla^{2} \mathbf{v}+\frac{1}{\rho_{f}} \nabla \mathrm{q}=-\frac{1}{\rho_{f}} \mathbf{D}_{p}\left(t-\epsilon_{R}\right) g\left[\mathbf{x}-\mathbf{x}_{p}\left(t-\epsilon_{R}\right), \epsilon_{R}\right]
$$

with the initial condition $\mathbf{v}\left(\mathbf{x}, t_{n}\right)=0$. For the sake of simplicity let us consider an Euler-like time integration algorithm. In order to achieve the solution $\mathbf{v}\left(\mathbf{x}, t_{n+1}\right)$ the operator in 92 is successively splitted into three steps, namely the forcing step, the diffusion step and the projection step which enforces the condition $\nabla \cdot \mathbf{v}=0$. Actually the forcing step gives

$$
\tilde{\mathbf{v}}\left(\mathbf{x}, t_{n+1}\right)=-\frac{\Delta t}{\rho_{f}} \mathbf{D}_{p}\left(t_{n}-\epsilon_{R}\right) g\left[\mathbf{x}-\mathbf{x}_{p}\left(t_{n}-\epsilon_{R}\right), \epsilon_{R}\right] .
$$

The diffusion step is readily achieved thanks to the semigroup property of solutions of the heat equation and the property (20), namely

$$
\mathbf{v}_{*}\left(\mathbf{x}, t_{n+1}\right)=\int \tilde{\mathbf{v}}\left(\boldsymbol{\xi}, t_{n+1}\right) g(\mathbf{x}-\boldsymbol{\xi}, \Delta t) d \boldsymbol{\xi},
$$


which results in the pseudo-velocity

$$
\mathbf{v}_{*}\left(\mathbf{x}, t_{n+1}\right)=-\frac{\Delta t}{\rho_{f}} \mathbf{D}_{p}\left(t_{n}-\epsilon_{R}\right) g\left[\mathbf{x}-\mathbf{x}_{p}\left(t_{n}-\epsilon_{R}\right), \epsilon_{R}+\Delta t\right] .
$$

The divergence-free solution is achieved in terms of the decomposition $\mathbf{v}\left(\mathbf{x}, t_{n+1}\right)=$ $\mathbf{v}_{*}\left(\mathbf{x}, t_{n+1}\right)+\nabla \Phi$ and the projection step $\nabla^{2} \phi=-\nabla \cdot \mathbf{v}_{*}$. By using the expression (95), after some algebra, the solution $\mathbf{v}\left(\mathbf{x}, t_{n+1}\right)$ can be evaluated in a closed form as

$\mathbf{v}\left(\mathbf{x}, t_{n+1}\right)=\frac{1}{\left(2 \pi \sigma^{2}\right)^{3 / 2}}\left\{\left[e^{-\eta^{2}}-\frac{f(\eta)}{2 \eta^{3}}\right] \mathbf{D}^{n}-\left(\mathbf{D}^{n} \cdot \hat{\mathbf{r}}\right)\left[e^{-\eta^{2}}-\frac{3 f(\eta)}{2 \eta^{3}}\right] \hat{\mathbf{r}}\right\}$.

In the above expression we have defined $\mathbf{D}^{n}=\mathbf{D}\left(t_{n}-\epsilon_{R}\right), \mathbf{r}=\mathbf{x}-\mathbf{x}_{p}\left(t_{n}-\epsilon_{R}\right)$, the hat denotes $\hat{\mathbf{r}}=\mathbf{r} / r, \eta=r / \sqrt{2} \sigma$ is the dimensionless distance with $\sigma=\sqrt{2 \nu\left(\epsilon_{R}+\Delta t\right)}$ and $f(\eta)=\frac{\sqrt{\pi}}{2} \operatorname{erf}(\eta)-\eta e^{-\eta^{2}}$.

\section{References}

[1] Balachandar, S. 2009 A scaling analysis for point-particle approaches to turbulent multiphase flows. International Journal of Multiphase Flow 35 (9), 801-810.

[2] Balachandar, S. \& Eaton, J.K. 2010 Turbulent dispersed multiphase flow. Ann. Rev. Fluid Mech 42, 111-133.

[3] Batchelor, George Keith 2000 An introduction to fluid dynamics. Cambridge university press.

[4] Bec, J., Biferale, L., Cencini, M., Lanotte, A. \& Toschi, F. 2010 Intermittency in the velocity distribution of heavy particles in turbulence. J. Fluid Mech 646, 527-536.

[5] Boivin, M., Simonin, O. \& Squires, K.D. 1998 Direct numerical simulation of turbulence modulation by particles in isotropic turbulence. Journal of Fluid Mechanics 375, 235-263.

[6] Burton, T.M. \& Eaton, J.K. 2005 Fully resolved simulations of a particle-turbulence interaction. J. Fluid Mech. 545, 67-111.

[7] Cate, A. Ten, Derksen, J.J., Portela, L.M. \& Akken, H.E.A. VAN DEN 2004 Fully resolved simulations of colliding monodisperse spheres in forced isotropic turbulence. J. Fluid Mech. 519, 233-271. 
[8] Climent, Eric \& Magnaudet, Jacques 2006 Dynamics of a twodimensional upflowing mixing layer seeded with bubbles: Bubble dispersion and effect of two-way coupling. Physics of Fluids (1994-present) 18 (10), 103304.

[9] Crowe, C.T., Sharma, M.P. \& Stock, D.E. 1977 The particlesource in cell method for gas droplet flow. J. Fluid Eng. 99, 325.

[10] Dance, S.L. \& Maxey, M.R. 2003 Incorporation of lubrication effects into the force-coupling method for particulate two-phase flow. J. Comp. Phys. 189, 212-238.

[11] Eckhardt, B. \& Buehrle, J. 2008 Time-dependent effects in high viscosity fluid dynamics. European Physical Journal 157, 135-148.

[12] Elgobashi, S. 2006 An updated classification map of particle-laden turbulent flows. In IUTAM Symposium on Computational Approaches to Multiphase Flow, pp. 3-10. Springer.

[13] Gao, H., Li, H. \& WANG, L.-P. 2011 Lattice boltzmann simulation of turbulent flow laden with finite-size particles. Computers \& Mathematics with Applications .

[14] Gatignol, R. 1983 The faxén formulas for a rigid particle in an unsteady non-uniform stokes-flow. Journal de Mécanique théorique et appliquée 2 (2), 143-160.

[15] Gualtieri, P, Casciola, CM, Benzi, R, Amati, G \& Piva, R 2002 Scaling laws and intermittency in homogeneous shear flow. Physics of Fluids (1994-present) 14 (2), 583-596.

[16] Gualtieri, P., Picano, F. \& Casciola, C.M. 2009 Anisotropic clustering of inertial particles in homogeneous shear flow. J. Fluid Mech. 629, 25-39.

[17] Gualtieri, P., Picano, F., Sardina, G. \& Casciola, C.M. 2013 Clustering and turbulence modulation in particle-laden shear flow. $J$. Fluid Mech. 715, 134-162.

[18] Homann, H. \& Bec, J. 2010 Finite-size effects in the dynamics of neutrally buoyant particles in turbulent flow. J. Fluid Mech. 651 (1), 81-91. 
[19] Jenny, Patrick, Roekaerts, Dirk \& Beishuizen, Nijso 2012 Modeling of turbulent dilute spray combustion. Progress in Energy and Combustion Science.

[20] Kim, S. \& Karilla, S.J. 2005 Microhydrodynamics. Dover publications, Inc.

[21] Lamb, Horace 1993 Hydrodynamics. Cambridge University Press.

[22] Liu, D., Keaveny, E.E., Maxey, M.R. \& Karniadakis, G.E. 2009 Force-coupling method for flows with ellipsoidal particles. Journal of Computational Physics 228 (10), 3559-3581.

[23] Lomholt, S. \& Maxey, M.R. 2003 Force-coupling method for particulate two phase flow: Stokes flow. J. Comp. Phys. 184, 381-405.

[24] Lucci, F., Ferrante, A. \& Elghobashi, S. 2010 Modulation of isotropic turbulence by particles of taylor length-scale size. J. Fluid Mech. 650 (1), 5-55.

[25] Marchioli, C. \& Soldati, A. 2002 Mechanisms for particle transfer and segregation in a turbulent boundary layer. J. Fluid. Mech. 468, 283 .

[26] Maxey, M.R. \& Patel, B.K. 2001 Localized force representations for particles sedimenting in stokes flow. Int. J. Mult. Flow 27, 1603-1626.

[27] Maxey, M.R. \& Riley, J.J. 1983 Equation of motion for a small rigid sphere in a nonuniform flow. Phys. Fluids 26, 2437.

[28] Meneguz, Elena \& Reeks, Michael W 2011 Statistical properties of particle segregation in homogeneous isotropic turbulence. Journal of Fluid Mechanics 686, 338-351.

[29] Monchaux, Romain, Bourgoin, Mickä̈l \& Cartellier, Alain 2010 Preferential concentration of heavy particles: a voronoi analysis. Physics of Fluids (1994-present) 22 (10), 103304.

[30] Naso, A. \& Prosperetti, A. 2010 The interaction between solid particle and turbulent flow. New J. of Phys. 12, 1-20.

[31] PAN, Y. \& BAnerJeE, S. 2001 Numerical simulation of particle interaction with wall turbulence. Phys. Fluids 8(10), 2733-2755. 
[32] Pasquetti, R, Bwemba, R \& Cousin, L 2008 A pseudo-penalization method for high reynolds number unsteady flows. Applied Numerical Mathematics 58 (7), 946-954.

[33] PaWlowski, L. 2008 The science and engineering of thermal spray coatings. Wiley.

[34] Picano, F., Sardina, G. \& Casciola, C.M. 2009 Spatial development of particle-laden turbulent pipe flow. Phys. Fluids 21, 093305.

[35] Pignatel, F., Nicolas, M. \& Guazzelli, E. 2011 A falling cloud of particles at a small but finite reynolds number. Journal of Fluid Mechanics 671, 34-51.

[36] Post, S.L. \& Abraham, J. 2002 Modeling the outcome of drop-drop collisions in diesel sprays. Int. J. Mult. Flows 28 (6), 997-1019.

[37] Reade, Walter C \& Collins, Lance R 2000 Effect of preferential concentration on turbulent collision rates. Physics of Fluids (1994present) 12 (10), 2530-2540.

[38] Rogallo, RS 1981 Numerical experiments in homogeneous turbulence. NASA, TM 81315 .

[39] Saffman, P.G. 1992 Vortex dynamics. Cambridge Univ Pr.

[40] Stone, Howard A, Stroock, Abraham D \& Ajdari, Armand 2004 Engineering flows in small devices: microfluidics toward a lab-ona-chip. Annu. Rev. Fluid Mech. 36, 381-411.

[41] Subramanian, G. \& Koch, D.L. 2008 Evolution of clusters of sedimenting low-reynolds-number particles with oseen interactions. Journal of Fluid Mechanics 603 (1), 63-100.

[42] Toschi, Federico \& Bodenschatz, Eberhard 2009 Lagrangian properties of particles in turbulence. Annual Review of Fluid Mechanics 41, 375-404.

[43] Yeo, K., Dong, S., Climent, E. \& Maxey, M.R. 2010 Modulation of homogeneous turbulcence seeded with finite size bubbles or particles. Int. J. Mult. Flow 36(3), 221-233.

[44] Zapryanov, Z \& Tabakova, S 1998 Dynamics of bubbles, drops and rigid particles, , vol. 50. Springer. 
[45] Zhang, Z. \& Prosperetti, A. 2005 A second order method for three dimensional particle simulation. J. Comp. Phys. 210, 292-324. 